\title{
Extremely primitive groups
}

\author{
Avinoam Mann, Cheryl E. Praeger and Ákos Seress
}

\begin{abstract}
A primitive permutation group is called extremely primitive if a point stabilizer acts primitively on each of its orbits. We prove that finite extremely primitive groups are of affine type or almost simple. Moreover, we determine the affine type examples up to finitely many exceptions.
\end{abstract}

Mathematics Subject Classification (2000). 20B15.

Keywords. Primitive permutation groups.

\section{Introduction}

A primitive permutation group is called extremely primitive if a point stabilizer is primitive on each of its orbits (where we regard the trivial group acting on a set of size 1 as primitive). Extremely primitive groups were the subject of a 1927 paper of W. A. Manning [22], and interest again focused on them following efforts to extend the Bounded Subdegrees Theorem proved by G. Schlichting [26] in 1980 (and reproved by V. Trofimov in 1985, and G. M. Bergman and H. W. Lenstra in 1989, see [23]) concerning the structure of a transitive permutation group given a finite upper bound on its subdegrees. Extremely primitive groups are among the most natural primitive groups to be studied further, and examples of these include

(a) $G \cong Z_{p}$ acting regularly on $\Omega=G$ by right multiplication, where $p$ is a prime, and

(b) each 2-primitive permutation group $G$ on $\Omega$; a classification of all such groups is available and depends on the finite simple group classification.

If a finite group $G$ is extremely primitive and is not one of these examples, then $G$ is not regular on $\Omega$, since the only primitive regular permutation groups are cyclic of prime order. Also $G$ is simply primitive, that is, $G$ is not 2-transitive, since the only

The second and third authors wish their colleague Avinoam Mann a happy and productive retirement. This project forms part of an Australian Research Council Discovery Grant of the second author. The third author was partially supported by the NSA and NSF. 
2-transitive examples are 2-primitive. Thus if we exclude these examples, then all finite extremely primitive groups $G$ satisfy the following conditions.

(A) $G$ is a simply primitive permutation group on a finite set $\Omega$ such that, for $\alpha \in \Omega$, the stabilizer $G_{\alpha} \neq 1$, and $G_{\alpha}$ acts primitively on each of its orbits in $\Omega$.

These conditions may be stated in an equivalent form as follows (setting $H:=G_{\alpha}$ ).

(A') $G$ is a finite group with a maximal non-trivial core-free subgroup $H$, such that $H \cap H^{x}$ is maximal in $H$, for each $x \in G \backslash H$, and there are at least three $H$-double-cosets.

It follows from an old result of Manning [22] (or see [30, 17.6]) that $G_{\alpha}$ is faithful on each of its orbits in $\Omega \backslash\{\alpha\}$. In the setting of $\left(\mathrm{A}^{\prime}\right)$, Manning's result is that $H \cap H^{x}$ is core-free in $H$ for each $x \in G \backslash H$. We study groups satisfying these conditions in an attempt to classify them, a problem that was mentioned in [23]. In that paper the authors considered transitive groups, not necessarily finite, with a point stabilizer acting primitively on each suborbit. As was explained there, the problem does not reduce to the case of primitive groups discussed here, but still that case seems a natural first step. We also confine ourselves to finite groups.

Our main results are as follows.

Theorem 1.1. If $G \leq \operatorname{Sym}(\Omega)$ is extremely primitive then $G$ is of affine type or almost simple.

The bulk of this paper is the further analysis of the affine case. We shall return to the classification of extremely primitive groups of almost simple type in a sequel. The proof of Theorem 1.1 is independent of the finite simple group classification, but further results rely on this classification: namely Theorem 1.2 through its use of the classification of finite 2-transitive groups, and Theorem 1.3 where more detailed information about simple groups and their representations is needed.

Theorem 1.2. Let $G \leq \operatorname{Sym}(\Omega)$ be extremely primitive of affine type, so that $|\Omega|=$ $p^{d}$ for some prime $p, G=N H \leq \operatorname{AGL}(d, p)$ with $N=Z_{p}^{d}$ and $H$ an irreducible subgroup of $\mathrm{GL}(d, p)$. Then one of the following holds.

(a) (Soluble examples)

(i) $d=1$ and $H=1$; or

(ii) $H=Z_{q}$, where $q$ is a prime, and $o(p \bmod q)=d$; or

(iii) $H=Z_{q} \cdot Z_{e}$, with $q$ as in (ii), and e divides $d$.

(b) (2-transitive, insoluble examples) $p=2$ and one of the following holds.

(i) $H=\operatorname{SL}(d, 2)$ for $d \geq 3$, or $H=\operatorname{Sp}(d, 2)$ for $d \geq 4$ even;

(ii) $(d, H)=\left(4, A_{6}\right),\left(4, A_{7}\right),(6, \operatorname{PSU}(3,3)),(6, \operatorname{PSU}(3,3) .2)$. 
(c) (Insoluble, simply primitive examples) $p=2$ and $H$ is almost simple.

Moreover each of the groups in parts (a) and (b) is extremely primitive.

To classify the finite affine extremely primitive groups it remains to find all the examples in part (c) of Theorem 1.2. This we do up to a finite number of possibilities.

Theorem 1.3. For the pairs $(d, H)$ in (a)-(c) below, the group $Z_{2}^{d} \cdot H$ is simply primitive and extremely primitive.

(a) $\operatorname{Soc}(H)$ sporadic:

(10, $\left.M_{12}\right)$, (10, $\left.M_{22}\right),\left(10, M_{22} .2\right),\left(11, M_{23}\right)$ (two groups), $\left(11, M_{24}\right)$ (two groups), $\left(22, \mathrm{Co}_{3}\right),\left(24, \mathrm{Co}_{1}\right)$;

(b) $\operatorname{Soc}(H)$ alternating:

$\left(2 k, A_{2 k+1}\right),\left(2 k, S_{2 k+1}\right)$ for $k \geq 2,\left(2 k, A_{2 k+2}\right),\left(2 k, S_{2 k+2}\right)$ for $k \geq 3$;

(c) $\operatorname{Soc}(H)$ of Lie type:

$\left(2 k, \Omega^{ \pm}(2 k, 2)\right),\left(2 k, \Omega^{ \pm}(2 k, 2) .2\right)$ for $k \geq 3,(8, \operatorname{PSL}(2,17)),(8, \operatorname{Sp}(6,2))$.

Moreover, there are only finitely many insoluble, simply primitive, extremely primitive groups of affine type not occurring on this list.

Note that up to permutational isomorphism there are two extremely primitive groups with structure $Z_{2}^{11} \cdot M_{23}$ and also there are two such groups with structure $Z_{2}^{11} . M_{24}$. We conjecture that the list in Theorem 1.3 is complete. We say more about this conjecture in Subsection 4.1. In particular, modulo a proof of a conjecture of G. E. Wall, the only possible additional examples are for the pairs $(d$, $\operatorname{Soc}(H))$ listed in Table 2 (see Theorem 4.8).

We prove Theorem 1.1 in Section 2, Theorem 1.2 in Section 3, and Theorem 1.3 in Section 4.

\section{Reduction to the affine and almost simple cases}

In this section we prove Theorem 1.1. If $G \leq \operatorname{Sym}(\Omega)$ is either cyclic of prime order or 2-transitive, then it is primitive of affine or almost simple type and there is nothing to prove. Hence it is enough to consider the case when $G, \Omega$, and $H:=G_{\alpha}$ satisfy condition (A), or equivalently, condition $\left(\mathrm{A}^{\prime}\right)$.

Let $N$ be a minimal normal subgroup of $G$. Then $N$ is transitive on $\Omega$ and hence $G=N H$. The various possibilities for the structure of $N$ and $H$ are described by the O'Nan-Scott Theorem [4, 4.1A]. First we treat the case where $N$ is regular on $\Omega$.

Lemma 2.1. If $N$ is regular on $\Omega$, that is, if $N \cap H=1$, then $N$ is elementary abelian, and hence Theorem 1.1 holds. 
Proof. We may identify $N$ with $\Omega$, so that $N$ acts on itself by right multiplication, and $H$ acts on $N$ by conjugation. Thus if $1 \neq x \in N$ and $h \in H \cap H^{x}$, then $h=h_{1}^{x}$ for some $h_{1} \in H$, so $\left[h_{1}, x\right]=h_{1}^{-1} h \in H \cap N=1$. Hence $h_{1} \in C_{H}(x)$, implying that $h=h_{1}$, and that $C_{H}(x)=H \cap H^{x}$. Therefore, $C_{H}(x)$ is maximal in $H$. Note that $C_{H}(x) \neq H$, because $H$ fixes only one point of $\Omega$.

Suppose that $N$ is not elementary abelian. Then $N$ is not nilpotent, $G$ is not a Frobenius group (see [30, Theorem $\left.5.1^{\prime}\right]$ ), and therefore some non-identity element $u \in \Omega=N$ is fixed by some non-identity element of $H$. Thus $C_{H}(u) \neq 1$, and it follows that $H$ is not of prime order. Then, for any $x \in N^{\#}$, the stabilizer $C_{H}(x)$ of $x$ in $H$ is maximal in $H$, and hence $C_{H}(x) \neq 1$.

Let $K_{1}, \ldots, K_{r}$ be the distinct subgroups of $H$ occurring as centralizers of nonidentity elements of $N$, with $K_{i}=C_{H}\left(x_{i}\right)$, say, and let $C_{i}=C_{N}\left(K_{i}\right)$. Now each element of $N$ lies in at least one of the subgroups $C_{i}$. If $i \neq j$, then $\left\langle K_{i}, K_{j}\right\rangle=H$ since $K_{i}, K_{j}$ are maximal in $H$ and so $C_{i} \cap C_{j}$ centralizes $\left\langle K_{i}, K_{j}\right\rangle=H$. Hence $C_{i} \cap C_{j}=1$. Thus the $C_{i}^{\#}=C_{1} \backslash\{1\}$ form a partition of $N^{\#}$. The insoluble groups with a partition were determined by M.Suzuki [28], and are the groups $\operatorname{PSL}(2, q)$, $\operatorname{Sz}(q)$, and $\operatorname{PGL}(2, q)$. Here $N$ must be one of the first two as it is minimal normal in $G$. If $N$ is the unique minimal normal subgroup of $G$, then $G$ is almost simple. Otherwise $G$ has exactly two minimal normal subgroups, which are isomorphic simple groups, say $N_{1}$ and $N_{2}$, two copies of the simple group $N$. Let $S=N_{1} \times N_{2}$, the socle of $G$. Then we may assume that $H \cap S$ is the diagonal subgroup $D=\{(x, x) \mid$ $x \in N\}$. Since $H$ is primitive on the $G$-conjugacy classes contained in $N_{1}$, and $D \triangleleft H, D$ is transitive on the same classes, which means that the $G$-classes are $N_{1^{-}}$classes. However, an outer automorphism of a non-abelian simple group moves some conjugacy class [5, Theorem $\mathrm{C}]$, and therefore $G$ induces only inner automorphisms on $N_{1}$, which in turn means that $G=N_{1} C_{G}\left(N_{1}\right)=S$ and $H=D$. However if $x \in N_{1}$ is a non-identity element of prime order $p$, where $p$ divides $q$, then $C_{H}(x)=\left\{(y, y) \mid y \in C_{N}(x)\right\}$ is not maximal in $H$, which is a contradiction.

Thus we may assume that $G$ has no regular minimal normal subgroups. By Theorem [4, 4.1A], $G$ has a unique minimal normal subgroup $N=T_{1} \times \cdots \times T_{k}$, where the $T_{i}$ are all isomorphic to a non-abelian simple group $T$, and $k \geq 1$. We show next that $G$ does not preserve a non-trivial Cartesian decomposition of the point set $\Omega$.

Lemma 2.2. There is no non-trivial decomposition $\Omega=\Sigma^{l}(l \geq 2)$ such that $G \leq \operatorname{Sym}(\Sigma)$ ? $S_{l}$ in product action.

Proof. Suppose that $\Omega=\Sigma^{l}(l \geq 2)$ and $G \leq \operatorname{Sym}(\Sigma)$ \& $S_{l}$ in product action. Since $N$ is non-abelian and non-regular, it follows from $[14,2.4]$ that, replacing $G$ by a conjugate in $\operatorname{Sym}(\Sigma)$ ? $S_{l}$ if necessary, we may assume that $G \leq L$ २ $S_{l}$ where $L$ is a primitive subgroup of $\operatorname{Sym}(\Sigma)$ and $N=\operatorname{Soc}(L)^{l}$. Thus $\operatorname{Soc}(L) \cong T^{m}$ where 
$k=l m$. Since $G=N H$, the subgroup $H$ is transitive on both the $k$ simple direct factors of $N$, and the $l$ entries of points of $\Sigma^{l}$.

We may take the point $\alpha \in \Omega$ to be $\alpha=(\sigma, \ldots, \sigma)$ for some $\sigma \in \Sigma$. Then $H=G_{\alpha} \leq\left(L \nmid S_{l}\right)_{\alpha}=L_{\sigma} \nmid S_{l}$. In particular, $N_{\alpha}=\left(\operatorname{Soc}\left(L_{\sigma}\right)\right)^{l}$, and since $N$ is not regular, $N_{\alpha} \neq 1$. Let $\Delta$ be an orbit for $H$ in $\Omega \backslash\{\alpha\}$. Then $N_{\alpha}=N \cap H$ is a nontrivial normal subgroup of $H$, and as $H$ acts faithfully and primitively on $\Delta$, it follows that $N \cap H$ is transitive on $\Delta$. Hence $H$ and $N \cap H$ have the same orbits in $\Omega$. Now the set $\{(\tau, \sigma, \sigma, \ldots, \sigma) \mid \tau \in \Sigma\}=\Sigma \times\{\sigma\}^{l-1}$ is clearly invariant under the action of $N \cap H$, and hence is a union of orbits of $N \cap H$. However as $H$ acts transitively on the entries of points of $\Sigma^{l}$, and $H \leq L_{\sigma} 2 S_{l}$, this subset is not invariant under the action of $H$, and hence is not a union of $H$-orbits.

It follows from Lemma 2.2 and the O'Nan-Scott Theorem (see [19]) that, if $G$ has no regular normal subgroup, and $G$ does not preserve a non-trivial Cartesian decomposition of $\Omega$, then either $G$ is almost simple, or $G$ has simple diagonal type. Thus in order to complete the proof of Theorem 1.1, it is sufficient to show that $G$ does not have simple diagonal type. Suppose to the contrary that it does. Then, replacing $G$ by a conjugate if necessary, we may assume that $G$ is a subgroup of the group $W$ defined by

$$
W=\left\{\begin{array}{l|l}
\left(a_{1}, \ldots, a_{k}\right) \cdot \pi & \begin{array}{l}
a_{i} \in \operatorname{Aut}(T), \pi \in S_{k}, \\
a_{i} \equiv a_{j}(\bmod \operatorname{Inn}(T))
\end{array} \text { for all } i, j
\end{array}\right\}
$$

where $\pi^{-1}\left(a_{1}, \ldots, a_{k}\right) \pi=\left(a_{1 \pi^{-1}}, \ldots, a_{k \pi^{-1}}\right)$. The socle of $W$ is the group $\operatorname{Soc}(W)=\left\{\left(t_{1}, \ldots, t_{k}\right) \mid t_{i} \in \operatorname{Inn}(T)\right\}$, the set $\Omega=T^{k-1}$, and $W$ acts on $\Omega$ as follows:

$$
\begin{aligned}
\left(a_{1}, \ldots, a_{k}\right):\left(t_{1}, \ldots, t_{k-1}\right) & \mapsto\left(a_{k}^{-1} t_{1} a_{1}, \ldots, a_{k}^{-1} t_{k-1} a_{k-1}\right) \\
\pi:\left(t_{1}, \ldots, t_{k-1}\right) & \mapsto\left(t_{k \pi^{-1}}^{-1} t_{1 \pi^{-1}}, \ldots, t_{k \pi^{-1}} t_{(k-1) \pi^{-1}}\right)
\end{aligned} \quad \text { and }
$$

for $\left(a_{1}, \ldots, a_{k}\right) \pi \in W$ and $\left(t_{1}, \ldots, t_{k-1}\right) \in T^{k-1}$, where $t_{k}=1_{T}$. Thus for $\alpha=\left(1_{T}, \ldots, 1_{T}\right) \in T^{k-1}, W_{\alpha}=A \times S_{k}$ where $A=\{(a, \ldots, a) \mid a \in \operatorname{Aut}(T)\}$. A subgroup $G$ of $W$ containing $N$ is primitive on $\Omega$ provided that $G$ acts primitively on the simple direct factors of $N$. Since $G=N H$ we have that $H$ acts primitively on these simple direct factors.

Lemma 2.3. If $G$ is of simple diagonal type, then $k=2$ and, for each $t \in T$, $t \neq 1$, there is an automorphism $a(t) \in \operatorname{Aut}(T)$ which inverts $t$ such that $a(t)^{2} \in$ $\operatorname{Inn}(T)$, and $\left\langle C_{\operatorname{Inn}(T)}(t), a(t)\right\rangle$ is a maximal subgroup of $\langle\operatorname{Inn}(T), a(t)\rangle$. Moreover, $\langle\operatorname{Inn}(T), a(t)\rangle$ is independent of $t \in T \backslash\{1\}$.

Proof. Suppose that $G$ is a subgroup of the group $W$ defined above. Consider the point $\delta=(t, 1, \ldots, 1) \in \Omega=T^{k-1}$, where $t \in T, t \neq 1$. The $N_{\alpha}$-orbit containing $\delta$ 
is the set $\left\{\left(t^{x}, 1, \ldots, 1\right) \mid x \in T\right\}$, which in particular has size greater than 1. Let $\Delta$ denote the $H$-orbit containing $\delta$. Since the normal subgroup $N_{\alpha}$ of $H$ acts nontrivially on $\Delta$, and since $H$ is primitive on $\Delta$ it follows that $\Delta=\left\{\left(t^{x}, 1, \ldots, 1\right) \mid x \in T\right\}$. However, since $H$ is transitive on the simple direct factors of $N$, it follows from the definition of the action that, when $k \geq 3$, the $H$-orbit containing $\delta$ contains a $(k-1)$-tuple with non-trivial second entry.

Therefore $k=2$, so $\Omega=T$ and $\Delta$ is the conjugacy class containing $t$. We have $N_{\alpha} \leq H \leq \operatorname{Aut}(T) \times\langle\tau\rangle$ where $\langle\tau\rangle=S_{2}$ and $\tau: x \mapsto x^{-1}$ for all $x \in T$. Now $H \cap \operatorname{Aut}(T)$ must leave the $H$-orbit $\Delta$ invariant, and it follows that $H \cap \operatorname{Aut}(T)$ leaves all conjugacy classes of $T$ invariant. Since for each outer automorphism of a non-abelian simple group $T$ there is a conjugacy class of $T$ which it moves [5, Theorem C], it follows that $H \cap \operatorname{Aut}(T)=\operatorname{Inn}(T)=N_{\alpha}$, and $\left|H: N_{\alpha}\right| \leq 2$.

Now $H$ contains an element which interchanges the two simple factors of $N$, so $\left|H: N_{\alpha}\right|=2$. Such an element is of the form $a \tau$ for some $a \in \operatorname{Aut}(T)$, and $a \tau: t \mapsto\left(t^{a}\right)^{-1}$. Since $N_{\alpha}$ is transitive on $\Delta$ we have $H=N_{\alpha} H_{\delta}$, so we may assume in addition that $a \tau \in H_{\delta}$. Therefore $t^{a}=t^{-1}$, and hence every element $t$ of $T$ is mapped to its inverse by some automorphism $a=a(t)$. Also $(a \tau)^{2}=a^{2} \in$ $H \cap \operatorname{Aut}(T)=\operatorname{Inn}(T)$. Now $H=\langle\operatorname{Inn}(T), a \tau\rangle$, and $H_{\delta}=\left\langle C_{\operatorname{Inn}(T)}(t), a \tau\right\rangle$ is a maximal subgroup of $H$. Moreover $H \leq W_{\alpha}=A \times S_{k}$, and $H$ projects faithfully onto $A$ with image $\langle\operatorname{Inn}(T), a\rangle$. It follows that $\left\langle C_{\operatorname{Inn}(T)}(t), a\right\rangle$, the image of $H_{\delta}$ under this projection, is a maximal subgroup of $\langle\operatorname{Inn}(T), a\rangle$, and that $\langle\operatorname{Inn}(T), a\rangle$ is independent of the choice of $t$ in $T \backslash\{1\}$.

We now prove that there are no simple groups satisfying the conditions of Lemma 2.3, thereby completing the proof of Theorem 1.1.

Lemma 2.4. There is no simple group satisfying the conditions on the group $T$ given in Lemma 2.3.

Proof. Let $T$ be a finite non-abelian simple group, and identify $T$ with the subgroup $\operatorname{Inn}(T)$ of its automorphism group $\operatorname{Aut}(T)$. Suppose that $T$ satisfies the conditions of Lemma 2.3, that is, there is a subgroup $A$ of $\operatorname{Aut}(T)$, containing $T$ as a subgroup of index at most 2, such that, for each $t \in T, t \neq 1$, there exists $a(t) \in A$ with $t^{a(t)}=t^{-1}, a(t)^{2} \in T$, and $\left\langle C_{T}(t), a(t)\right\rangle$ maximal in $A$. Note that $a(t)$ normalizes $C_{T}(t)$, and that $\left|C_{T}(t)\langle a(t)\rangle: C_{T}(t)\right| \leq 2$. Index 1 occurs if and only if $t$ is an involution and $a(t) \in T$, that is, $A=T$. In any case, $C_{T}(t)$ is normal in $C_{T}(t)\langle a(t)\rangle$.

Let $t$ be an involution, and assume that some non-identity element $x \in C_{T}(t)$ has odd order. Then both $t$ and $x$ are powers of $t x$. Also $a(t x)$ inverts $x$ and fixes $t$, and $C_{T}(t x)$ fixes both $x$ and $t$. Hence $\left\langle C_{T}(x t), a(x t)\right\rangle \leq\left\langle C_{T}(t), a(t)\right\rangle=$ $C_{A}(t)$ and $C_{T}(t x) \leq C_{T}(x)$. The maximality condition of Lemma 2.3 implies that $\left\langle C_{T}(t x), a(t x)\right\rangle=C_{A}(t)$. It follows that $x$ centralizes a subgroup of $C_{T}(t)$ of index at most 2 , and therefore all elements of odd order in $C_{T}(t)$ lie in $Z\left(O^{2}\left(C_{T}(t)\right)\right)$ (where 
$O^{2}\left(C_{T}(t)\right)$ is the smallest normal subgroup of $C_{T}(t)$ with quotient a 2-group). Thus the set consisting of all these elements forms a normal 2-complement in $C_{T}(t)$. By D. Gorenstein's characterization [7] of groups of this type, $T$ is one of the simple groups PSL $(2, q), \operatorname{Sz}(q), \operatorname{PSL}(3,4)$, or $A_{7}$.

If $T$ is one of $\operatorname{PSL}(n, q)$ or $\operatorname{Sz}(q)$, let $t$ be an element of order $p$, where $p$ is the defining characteristic. In $A_{7}$, let $t=(123)$ (456). Write $C=C_{T}(t)$. Since $\langle C, a(t)\rangle \leq N_{A}(C)$, the maximality implies equality, and $\left|N_{A}(C): C\right| \leq 2$. For these groups, this is only possible if $A=T=\operatorname{PSL}(2,5)$, but for this group the condition fails on taking $t$ to be an involution.

\section{Affine groups}

In this section we prove Theorem 1.2. Throughout this section we shall assume that $G$ is extremely primitive of affine type, and is not regular of prime order. Thus we have $G=N H$, where $N=Z_{p}^{d}$ and $H$ is a non-trivial irreducible subgroup of $\operatorname{GL}(d, p)$, for some prime $p$ and positive integer $d$. The centralizer of $H$ in $\operatorname{GL}(d, p)$ is therefore isomorphic to $\operatorname{GF}\left(p^{a}\right)^{\#}$, for some integer $a$ dividing $d$ (possibly $a=1$ ). We may, and shall, assume that $H \leq \mathrm{GL}\left(d / a, p^{a}\right) \cdot a$, and identify $N$ with the additive group of a $(d / a)$-dimensional vector space $V$ over the field $F=\mathrm{GF}\left(p^{a}\right)$. The integer $a$ is the largest for which such an embedding is possible. Further we identify $\Omega=V$, with $N$ and $H$ acting naturally. First we examine elements of $H$ which fix some 1-dimensional subspace of $\Omega$.

Lemma 3.1. If $h \in H$ and $v \in \Omega$ are such that $v^{h}=\lambda v$ for some $\lambda \in F$, then $\lambda=1$ (that is, 1 is the only possible eigen-value for an element of $H$ ). In particular, $H$ contains no non-identity element of order dividing $p^{a}-1$.

Proof. Suppose that $0 \neq v \in \Omega$ and $h \in H$ are such that $v^{h}$ lies in the $F$-space $\langle v\rangle_{F}$ spanned by $v$. Then $\left\langle v^{h}\right\rangle_{F}=\langle v\rangle_{F}$. Moreover, identifying $v$ with the corresponding element of $N$, we have that $C_{H}(v)^{h}=C_{H}\left(v^{h}\right)$ fixes each element of $\left\langle v^{h}\right\rangle_{F}=\langle v\rangle_{F}$, and it follows that $C_{H}(v)^{h}=C_{H}(v)=H \cap H^{v}$. By condition (A), $H \cap H^{v}$ is maximal in $H$, and since $h$ normalizes $C_{H}(v)=H \cap H^{v}$, it follows that $h \in C_{H}(v)$, and therefore $v^{h}=v$. Thus 1 is the only possible $F$-eigen-value for any element of $H$. However, if the order of $h$ divides $p^{a}-1$, then all the eigen-values of $h$ (in its splitting field) lie in $F$, and it follows that the only such element is $h=1$.

We now consider the actions of normal subgroups of $H$, and then we deal with soluble extremely primitive groups.

Lemma 3.2. If $M$ is a non-trivial normal subgroup of $H$, then $M$ and $H$ have the same orbits in $\Omega$, and $M$ leaves invariant no proper non-trivial subgroup of $N$. 
Proof. As in the proof of Lemma 2.1, we denote by $K_{1}, \ldots, K_{s}$ the distinct intersections $H \cap H^{x}=C_{H}(x)$, for $x \in N^{\#}$. Then by Condition (A), each $K_{i}$ is a maximal subgroup of $H$. The fact that $H$ is faithful on each of its orbits implies that $M$ is not contained in any $K_{i}$, so $H=M K_{i}$ for each $i$. This is equivalent to the assertion that $M$ is transitive on each $H$-orbit in $\Omega \backslash\{0\}$. Suppose that $L$ is a non-identity $M$-invariant subgroup of $N$, and let $1 \neq x \in L$. Because $H$ is primitive on the $H$-orbit $x^{H}$ containing $x, M$ is transitive on $x^{H}$, and hence this orbit is contained in $L$. Thus $L$ is $H$-invariant, so $L=N$.

Lemma 3.3. If $G$ is soluble and extremely primitive, then part (a) of Theorem 1.2 holds.

Proof. If $H=1$ then case (a) (ii) of Theorem 1.2 holds, so we may take $H \neq 1$ (as assumed for this section). Let $M$ be a minimal normal subgroup of $H$. By Lemma 3.2, $M$ is transitive on each $H$-orbit. Since $H$ is soluble, $M$ is elementary abelian, and since a transitive abelian permutation group is regular, it follows that $M$ induces a regular permutation group on each $H$-orbit in $\Omega \backslash\{0\}$. Then since $H$, and hence also $M$, acts faithfully on each such $H$-orbit, it follows that each of these $H$-orbits has length $|M|$. Hence $G$ is a soluble $3 / 2$-transitive group. These groups were determined by Passman [24, 25]. The examples are divided into four categories, and we consider each category in turn, to determine which of the soluble 3/2-transitive groups satisfy Condition (A).

1. $G$ is a Frobenius group. In this case $N$ is the Frobenius kernel and $H$ is a Frobenius complement. The intersections $H \cap H^{x}\left(x \in N^{\#}\right)$ are trivial, so $H$ is primitive and regular on each of its orbits in $\Omega \backslash\{0\}$. Thus $H$ has prime order, say $q$. The maximality of $H$ in $G$ is equivalent to $N$ being a minimal normal subgroup, so $G$ is as in case (a) (ii) of Theorem 1.2.

2. $G \leq A \Gamma \mathrm{L}\left(1, p^{d}\right)$. Here $H \leq \Gamma \mathrm{L}\left(1, p^{d}\right)$, so $H$ is metacyclic, and if the cyclic normal subgroup $T=H \cap \operatorname{GL}\left(1, p^{d}\right)$ is trivial, then $H$ is cyclic. Choosing $M$, in the argument above, to be a subgroup of $T$ if $T \neq 1$, then $M$ has prime order, $q$ say, and we see that each $H$-orbit in $\Omega \backslash\{0\}$ has length $q$, and that $M$ is transitive on it. If $T=1$, then $H=M$, and case (a) (ii) of Theorem 1.2 holds. If $T \neq 1$, then $T$ is faithful and transitive on each of the $H$-orbits and hence $|T|=q$. If $T$ is contained in $\mathrm{GL}\left(1, p^{b}\right)$, for some proper subfield $\mathrm{GF}\left(p^{b}\right)$ of $F$, then this subfield constitutes a proper $H$-invariant subgroup of $N$, contradicting the maximality of $H$ in $G$. Thus $T$ is contained in no such subgroup, which means that $N T$ is as in case (a) (ii) of Theorem 1.2. In this case, we may identify $\Omega$ with the additive group of the field $F$. Let $K=K_{1}$ be the stabilizer in $H$ of the multiplicative identity 1 of $F=\Omega$. Then we have $K \cap T=1$, and $K$ is a subgroup of the Galois group of $F$. If $K=1$ then $G=N T$ is as in case (a) (ii) of Theorem 1.2, while if $K \neq 1$, then $K$ is cyclic of order $e$, where $e$ divides $d$, and $G$ is as in case (a) (iii) of Theorem 1.2. 
3. $G \leq A \Gamma \mathrm{L}(2, q)$, where $q=p^{d / 2}$ with $d$ even, $H \leq \Gamma \mathrm{L}(2, q)$, and $H \cap$ $\mathrm{GL}(2, q)$ consists of diagonal or anti-diagonal matrices of determinant 1 or -1 . (By an anti-diagonal matrix we mean one whose nonzero entries are all on the northeast to southwest diagonal.) In this case $H$ is the union of diagonal and anti-diagonal matrices, and so it is a dihedral group of order $2 q$, say. Its cyclic subgroup $T$ of index 2 acts faithfully and transitively on each of the $H$-orbits in $\Omega \backslash\{0\}$. Therefore, for each $x \in N^{\#}, H \cap H^{x}$ has order 2, and since $H \cap H^{x}$ is maximal in $H$ it follows that $q$ is prime. Again $G$ is as in case (a) (iii) of Theorem 1.2, with $e=2$.

4. $G$ is one of several exceptional groups, of degrees $3^{2}, 5^{2}, 7^{2}, 11^{2}, 17^{2}$, or $3^{4}$. We will show that this case does not lead to new examples. First, Lemma 3.1 shows that $|H|$ is odd. Since all $H$-orbits in $\Omega \backslash\{0\}$ have equal size, the indices $\left|H: H \cap H^{x}\right|\left(x \in N^{\#}\right)$ divide $p^{d}-1$. The only possible odd divisors are 3, 5, 15, and 9. Here 15 is impossible, because the index of a maximal subgroup in a soluble group is a prime-power. If the index is 3 or 5 , then $H$ is isomorphic to a subgroup of odd order of either $S_{3}$ or $S_{5}$, and it follows that $H$ itself has order 3 or 5 respectively. Then considering a minimal normal subgroup of $G$ the maximality of $H$ implies that $G$ is as in case (a) (ii) of Theorem 1.2. Finally let the index be 9. Then $|N|=17^{2}$, and since $H$ acts faithfully as a soluble and primitive permutation group of degree $9, H$ contains a normal elementary abelian subgroup of order 9 . However, $H \leq \Gamma \mathrm{L}(2,17)$, which has no elementary abelian subgroup of order 9 .

For the rest of this section we therefore assume that $H$ is insoluble. First, we verify that the list of 2-transitive extremely primitive groups in Theorem 1.2 is correct.

Lemma 3.4. If $G=Z_{p}^{d}$.H is insoluble and 2-transitive, then part (b) of Theorem 1.2 holds.

Proof. The 2-transitive affine permutation groups $G$ are known (see e.g. [2, Table 7.3]) and more details on the structure of $H$ can be found in [17, Appendix]. There are three infinite families, with $H$ containing a normal subgroup isomorphic to one of the following three types: $\operatorname{SL}\left(d / a, p^{a}\right)$ for some $a \leq d / 2, \operatorname{Sp}\left(d / a, p^{a}\right)$ for some $a \leq d / 4$, or $G_{2}\left(p^{a}\right)^{\prime}$ for $p=2$ and $a=d / 6$. There are also eleven sporadic examples with $2^{4} \leq p^{d} \leq 59^{2}$. If $H$ belongs to one of the three infinite families then $H$ contains elements of order $p^{a}-1$ and so, by Lemma 3.1, we have $p^{a}=2$. This leads to the examples $H=\operatorname{SL}(d, 2), \operatorname{Sp}(d, 2), \operatorname{Sp}(4,2)^{\prime} \cong A_{6}, G_{2}(2)^{\prime} \cong$ $\operatorname{PSU}(3,3)$, and $G_{2}(2) \cong \operatorname{PSU}(3,3) .2$ listed in Theorem 1.2 (b) (in the last two cases, we constructed $H$ in GAP [6] to verify that $H$ acts primitively on $\Omega \backslash\{\alpha\}$ ). Out of the eleven sporadic examples, in eight cases $H$ does not have a faithful primitive representation (either $\operatorname{Soc}(H)$ is not the product of isomorphic simple groups, or $|\operatorname{Soc}(H)|=2)$. The remaining three cases are $G=Z_{3}^{6} \cdot \operatorname{SL}(2,13), Z_{11}^{2} \cdot \operatorname{SL}(2,5)$, and $Z_{2}^{4} . A_{7}$. Lemma 3.1 eliminates the first two of these and the third one leads to an example listed in Theorem 1.2 (b). 
Finally, suppose that $G$ is uniprimitive and $H$ is insoluble. There are two types of information affecting the structure of $H$. On the one hand, $H$ is an absolutely irreducible subgroup of $\operatorname{GL}\left(d / a, p^{a}\right) . a$, and we have available the Aschbacher classification [1] of such subgroups to provide a framework for our investigation. On the other hand, $H$ acts faithfully as a primitive permutation group on each of its orbits in $\Omega \backslash\{0\}$, and the O'Nan-Scott Theorem (see [19]) therefore provides information about the possible structure of $H$. We use a combination of these methods to complete the proof of Theorem 1.2. First we exploit the fact that $H$ is primitive on each of its orbits in $\Omega \backslash\{0\}$ to prove that $H$ has a unique minimal normal subgroup which is non-abelian.

Lemma 3.5. If $G$ is insoluble, then $p=2$, and $H$ has a unique minimal normal subgroup $M$, and $M$ is nonabelian.

Proof. If $p$ were odd, then by Lemma 3.1, $|H|$ would be odd also, and hence $G$ would be soluble, which is not the case. Hence $p=2$. Let $M$ be a minimal normal subgroup of $H$. By Lemma 3.2, each $H$-orbit is also an $M$-orbit. Suppose that $M$ is regular on each non-trivial orbit of $H$. Then all these orbits have length $|M|$, and hence $|M|$ divides $|\Omega|-1=2^{d}-1$. Thus $|M|$ is odd, and so $M$ is soluble, and hence is elementary abelian. Also, since $H$ is faithful of each non-trivial orbit, $M$ is self-centralizing in $H$. By Lemma 3.2, $M$ leaves invariant no non-trivial proper subgroup of $N$. Hence $M$ is an abelian irreducible group on $N$, and therefore $M$ is cyclic. Then $H / M$, which is isomorphic to a group of automorphisms of $M$, is abelian and $H$ is soluble, which is a contradiction. Thus $M$ is not regular on some $H$-orbit, and because $H$ is faithful and primitive on that orbit, it follows (see [19]) that $M$ is not abelian and is the unique minimal normal subgroup of $H$.

We now apply the Aschbacher classification [1] of subgroups of $\operatorname{GL}(d, p)$ to complete the proof of Theorem 1.2. We use the notation $\ell_{1}-\ell_{8}, 8$ of [13] to denote the categories of this classification. Recall that $H \leq \mathrm{GL}\left(d / a, p^{a}\right) . a$ and $a$ is maximal with respect to this property. We view $H$ as acting semi-linearly on $V=\operatorname{GF}\left(p^{a}\right)^{d / a}$. By Lemma 3.5, $p=2$ and $H$ has a unique minimal normal subgroup $M$ that is non-abelian.

Let $H_{0}:=H \cap \mathrm{GL}\left(d / a, 2^{a}\right)$. If $H_{0} \geq \mathrm{SL}\left(d / a, 2^{a}\right)$, or $H_{0}$ contains a classical group of dimension $d / a$ over $\operatorname{GF}\left(2^{a}\right)$, then Lemma 3.5 implies that $H_{0}$ is almost simple, satisfying the conclusion of Theorem 1.2 (c), and moreover in this case $M=$ $\operatorname{Soc}(H)$ is absolutely irreducible on $V$. Thus we may assume that $H_{0}$ is contained in $\operatorname{GL}\left(d / a, 2^{a}\right) \cdot a$, or a general symplectic, general unitary or general orthogonal group of dimension $d / a$ over $\operatorname{GF}\left(2^{a}\right)$, but neither contains the socle mod scalars of this group, nor leaves invariant any additional nondegenerate form. Then [1] implies that $H_{0}$ is in category $\ell_{i}$ for some $i \leq 7$, or in category 8 , for this group. If $H_{0}$ is in $\delta$, 
then by Lemma 3.5, $H_{0}$ is almost simple, satisfying the conclusion of Theorem 1.2 (c), and by [1], $\operatorname{Soc}(H)$ is absolutely irreducible on $V$. Thus we have the following.

Lemma 3.6. If $H$ is insoluble and simply primitive, then either

(a) $H$ is almost simple satisfying Theorem $1.2(\mathrm{c})$, not realisable over a proper subfield, and $\operatorname{Soc}(H)$ is absolutely irreducible on $V=\mathrm{GF}\left(2^{a}\right)^{d / a}$, or

(b) $H_{0}$ is in category $\mathcal{C}_{i}$ for some $i \in\{1,2, \ldots, 7\}$.

We may therefore assume that $H_{0}$ is in category $C_{i}$ where $1 \leq i \leq 7$. By Lemma 3.2, $H_{0}$ leaves invariant no proper GF(2)-subspace of $V$. Hence $H_{0}$ is not a $\ell_{1}$-subgroup. Moreover, since $H_{0}$ is irreducible on $V$, the maximality of $a$ implies that $H_{0}$ is not a $\ell_{3}$-subgroup of $\operatorname{GL}\left(d / a, 2^{a}\right)$ and in particular $H_{0}$ is absolutely irreducible on $V$, considered as a $\operatorname{GF}\left(2^{a}\right)$-vector space. Suppose that there is a proper subfield $\operatorname{GF}(q)$ of $\operatorname{GF}\left(2^{a}\right)$ such that $H_{0}$ is conjugate in $\operatorname{GL}\left(d / a, 2^{a}\right)$ to a subgroup of $Z \circ \operatorname{GL}(d / a, q)$, where $Z$ is the subgroup of scalar matrices in $\operatorname{GL}\left(d / a, 2^{a}\right)$. Then $H_{0}$ leaves invariant a GF(2)-subspace of $V$ of order at most $\left(2^{a} / q\right) q^{d / a}<2^{d}$, contradicting Lemma 3.2. Thus the representation of $H_{0}$ is realizable over no proper subfield of $\mathrm{GF}\left(2^{a}\right)$ (that is, $H_{0}$ is not in category $\ell_{5}$ ). Also, by Lemma 3.5, it follows that $H_{0}$ is not contained in the normalizer of a symplectic type $r$-group, where $d / a$ is a power of the prime $r$ (category $\varrho_{6}$ ). It therefore follows from [1] that either Theorem 1.2 (c) holds, or we have one of the following.

(i) $H_{0}$ leaves invariant a direct sum decomposition $\Omega=U_{1} \oplus \cdots \oplus U_{t}$ where each $U_{i}$ is a $\operatorname{GF}\left(2^{a}\right)$-subspace of dimension $d / a t, t>1$, and $H_{0}$ acts transitively on $\left\{U_{1} \ldots, U_{t}\right\}$ (category $C_{2}$ ).

(ii) $H_{0}$ leaves invariant a tensor product decomposition $\Omega=U \otimes W$ where $U, W$ are $\operatorname{GF}\left(2^{a}\right)$-subspaces with $\operatorname{dim} U=m>1, \operatorname{dim} W=n>1$, and $d / a=n m$ (category $\boldsymbol{C}_{4}$ ).

(iii) $H_{0}$ leaves invariant a tensor imprimitivity system $\Omega=U_{1} \otimes \cdots \otimes U_{t}$, such that each $U_{i}$ is a $\operatorname{GF}\left(2^{a}\right)$-subspace of dimension $m>1, d / a=m^{t}>m$, and $H_{0}$ acts transitively on $\left\{U_{1} \ldots, U_{t}\right\}$ (category $C_{7}$ ).

In the next lemmas we prove that none of the cases (i)-(iii) holds, thereby completing the proof of Theorem 1.2.

Lemma 3.7. Case (i) above does not hold.

Proof. Suppose that (i) holds and let $u$ be a non-zero vector in $U_{1}$. By Lemma 3.2, $M, H_{0}, H$ have the same orbits in $\Omega$. Let $\Delta$ denote the $H$-orbit containing $u$. Then $U_{1} \cap \Delta$ is a block of imprimitivity for the action of $H$ on $\Delta$. Since $H_{0}$ permutes the $U_{i}$ transitively, $U_{1} \cap \Delta$ is a proper subset of $\Delta$, and since $H$ is primitive on $\Delta$ it follows that $U_{1} \cap \Delta=\{u\}$. This means that the sum $v$ of the vectors in $\Delta$ is a non-zero vector which is left invariant by $H$, contradicting the fact that $H$ is irreducible on $\Omega$. 
Lemma 3.8. Case (ii) above does not hold.

Proof. Suppose that (ii) holds, and let $u, w$ be non-zero vectors in $U, W$ respectively. Let $\Delta$ denote the $H$-orbit containing $u \otimes w$. Then $H$ acts primitively on $\Delta$. Set $U \otimes w:=\left\{z \otimes w \mid z \in U^{\#}\right\}$. Then $\Delta \cap(U \otimes w)$ contains $u \otimes w$ and is a block of imprimitivity for $H$ in $\Delta$. Moreover, $\Delta \cap(U \otimes w)$ is not equal to $\Delta$, for if it were then $H$, in its induced action on $W$, would fix $\langle w\rangle_{F}$, whereas $H$ is irreducible on $W$ and $\operatorname{dim} W>1$. It follows that $\Delta \cap(U \otimes w)=\{u \otimes w\}$. Now the stabilizer $K$ in $H$ of the 1-space $\langle w\rangle_{F}$ is equal to the setwise stabilizer in $H$ of $U \otimes w$. From what we have just shown, $K$ fixes $\Delta \cap(U \otimes w)=\{u \otimes w\}$. Thus $K=H_{\langle u\rangle_{F}}$. Since $u$ was any non-zero vector in $U$, it follows that $K$ fixes each 1 -space $\left\langle u^{\prime}\right\rangle_{F}, u^{\prime} \in U^{\#}$. Thus $K$ acts on $U$ as a subgroup of scalar matrices, and similarly $K=H_{\langle u\rangle_{F}}$ acts on $W$ as a subgroup of scalar matrices. Since, by Lemma 3.1, the only eigen-value for elements of $H$ is 1 , it follows that $K=1$. Thus $H$ acts faithfully, regularly and primitively on $\Delta$, whence $H$ is cyclic. This contradicts the assumption that $H$ is insoluble.

The proof that case (iii) does not hold is rather more delicate. We first standardise the representation of such groups.

Lemma 3.9. Suppose that case (iii) above holds. We may identify each of the $U_{i}$ with an m-dimensional $\mathrm{GF}\left(2^{a}\right)$-vectorspace $U$ in such a way that $H \leq(\mathrm{GL}(U) \circ$ $\ldots \circ \mathrm{GL}(U)) . S_{t}$, where $\circ$ denotes the central product. Let $K$ denote the subgroup of $\mathrm{GL}(U)$ induced on $U=U_{1}$ by the stabilizer $H_{1}$ in $H$ of the first tensor factor $U_{1}$. Then, replacing $H$ by a conjugate under $\mathrm{GL}(U) \circ \cdots \circ \mathrm{GL}(U)$ if necessary, we may assume that $H \leq\left(N_{\mathrm{GL}(U)}(K) \circ \cdots \circ N_{\mathrm{GL}(U)}(K)\right) . S_{t}$ and $H \cap(\mathrm{GL}(U) \circ \cdots \circ \mathrm{GL}(U)) \leq$ $K \circ \cdots \circ K$.

Proof. We work with the preimage of $H$ in $\operatorname{GL}(U) ? S_{t}$. By definition, $K=K_{1}$ is the set of all $h_{1} \in \mathrm{GL}(U)$ such that there exists $\left(h_{1}, h_{2}, \ldots, h_{t}\right) \pi \in H$ with $1 \pi=1$. The subgroups $K_{j}(j \leq t)$ of $\mathrm{GL}(U)$ induced on $U_{j}=U$ by the stabilizer $H_{j}$ in $H$ of the $j^{\text {th }}$ tensor factor $U_{j}$ are defined similarly.

Let $x \rho \in H$, where $x=\left(x_{1}, \ldots, x_{t}\right) \in \operatorname{GL}(U)^{t}$ and $\rho \in S_{t}$, and suppose that $i \rho=j$. We claim that $K_{i}^{x_{i}}=K_{j}$. Let $k \in K_{i}$. There exists $h \pi \in H$ such that $h_{i}=k$ and $i \pi=i$. Then $(h \pi)^{x \rho} \in H$, and

$$
(h \pi)^{x \rho}=h^{x \rho} \cdot\left(x^{-1}\right)^{\rho} \cdot(x)^{\pi^{-1} \rho} \cdot \pi^{\rho}=x^{\prime} \pi^{\rho} .
$$

Now $(j) \pi^{\rho}=j$, and the $j^{\text {th }}$ entry of $x^{\prime}$ is equal to the $i^{\text {th }}$ entry of $h^{x} \cdot x^{-1} \cdot x^{\pi^{-1}}$, and this is $h_{i}^{x_{i}} x_{i}^{-1} x_{i}=h_{i}^{x_{i}}=k^{x_{i}}$. Hence $k^{x_{i}} \in K_{j}$, and so $K_{i}^{x_{i}} \subseteq K_{j}$. Similarly, since $(x \rho)^{-1}=\left(x^{-1}\right)^{\rho} \rho^{-1} \in H$ and $j \rho^{-1}=i$, we have that $K_{j}^{x_{i}^{-1}} \subseteq K_{i}$. Hence $K_{i}^{x_{i}}=K_{j}$, as claimed. 
Since $H$ is transitive on $\left\{U_{1}, \ldots, U_{t}\right\}$, for each $i=1, \ldots, t$, there exists $x^{(i)} \rho^{(i)}$ in $H$ such that $1 \rho^{(i)}=i$, and consequently $K_{1}^{x_{1}^{(i)}}=K_{i}$. Set $x:=\left(x_{1}^{(1)}, x_{1}^{(2)} \ldots, x_{1}^{(t)}\right) \in$ $\operatorname{GL}(U)^{t}$. Then $H^{x^{-1}}$ induces the same transitive subgroup of $S_{t}$ on the $U_{j}$, and, for $i=1, \ldots, t$, the subgroup of GL $(U)$ induced by $\left(H^{x^{-1}}\right)_{i}$ on the $i^{\text {th }}$ tensor factor $U_{i}$ is $x_{1}^{(i)} K_{i}\left(x_{1}^{(i)}\right)^{-1}=K_{1}=K$. Thus, replacing $H$ by $H^{x^{-1}}$, we may assume that $K_{1}=\cdots=K_{t}=K$. This means that $H \cap \mathrm{GL}(U)^{t} \leq K^{t}$. Finally consider a typical element $x \rho$ in the replaced subgroup $H$. For each $i$, we have shown that $K_{i}^{x_{i}}=K_{i \rho}$, that is, $x_{i} \in N_{\mathrm{GL}(U)}(K)$.

Lemma 3.10. Case (iii) above, with $t \geq 3$, does not hold.

Proof. Suppose that (iii) holds, with $t \geq 3$. By Lemma 3.9, we may assume that $H \leq\left(N_{\mathrm{GL}(U)}(K) \circ \cdots \circ N_{\mathrm{GL}(U)}(K)\right) . S_{t}$ and $H \cap(\mathrm{GL}(U) \circ \cdots \circ \mathrm{GL}(U)) \leq K \circ \cdots \circ K$, where $N_{\mathrm{GL}(U)}(K)$ is an irreducible subgroup of $\mathrm{GL}(U)$. Let $\left\{e_{1}, \ldots, e_{m}\right\}$ be a basis for $U$. Then the set $\mathbb{B}$ of $m^{t}$ vectors $e_{i_{1}} \otimes \cdots \otimes e_{i_{t}}$ (where each $i_{j} \leq m$ ) forms a basis for $\Omega$. (Since we have identified each of the $U_{i}$ with $U$, the order in these tensor expressions matters.)

Consider the vector $v=e_{1} \otimes e_{1} \otimes \cdots \otimes e_{1}+e_{1} \otimes e_{2} \otimes \cdots \otimes e_{2}$. In this representation for $v$ as a sum of two basic tensors, the two summands have equal first tensor entries, and all the other pairs of tensor entries are linearly independent. Suppose that there is another representation of $v$ as

$$
v=u_{1} \otimes \cdots \otimes u_{t}+w_{1} \otimes \cdots \otimes w_{t}
$$

where the $u_{j}, w_{j} \in U_{j}$, and there exists an $i$ such that $\left\{u_{i}, w_{i}\right\}$ is linearly dependent, but for each $j \neq i,\left\{u_{j}, w_{j}\right\}$ is linearly independent. We claim that $i=1$. Suppose, for a contradiction, that this is not the case. Recall that $t \geq 3$. Without loss of generality, we may assume that $i=2$, and that $u_{2}=w_{2}$. For each $j$, let $u_{j}=$ $\sum_{l} c_{j l} e_{l}$ and $w_{j}=\sum_{l} d_{j l} e_{l}$ (so $c_{2 l}=d_{2 l}$ for all $l$ ). Then

$$
v=\sum_{\left(j_{1}, \ldots, j_{t}\right)}\left(\prod_{l=1}^{t} c_{l} j_{l}+\prod_{l=1}^{t} d_{l j_{l}}\right)\left(e_{j_{1}} \otimes \cdots \otimes e_{j_{t}}\right) .
$$

Equating the coefficients of $e_{1} \otimes e_{1} \otimes \cdots \otimes e_{1}$ in the two expressions for $v$ as linear combinations in the basis $\mathbb{B}$ gives

$$
1=\prod c_{l 1}+\prod d_{l 1}=c_{21}\left(\prod_{l \neq 2} c_{l 1}+\prod_{l \neq 2} d_{l 1}\right)=c_{21} \alpha,
$$

say. Therefore both $c_{21}$ and $\alpha$ are non-zero. Next, equating the coefficients of $e_{1} \otimes e_{2} \otimes e_{1} \otimes \cdots \otimes e_{1}$ gives $0=c_{22} \alpha$, whence $c_{22}=0$. Then, equating the 
coefficients of $e_{1} \otimes e_{2} \otimes \cdots \otimes e_{2}$ gives

$$
1=c_{22}\left(c_{11} \prod_{l \geq 3} c_{l 2}+d_{11} \prod_{l \geq 3} d_{l 2}\right),
$$

which contradicts the fact that $c_{22}=0$. Thus in any such expression for $v$, the value of $i$ is 1 .

Let $\Delta$ denote the $H$-orbit containing $v$, and note that by assumption $H$ is primitive on $\Delta$. Each vector $z$ in $\Delta$ can be expressed as $z=u_{1} \otimes \cdots \otimes u_{t}+w_{1} \otimes \cdots \otimes w_{t}$, where the $u_{j}, w_{j} \in U_{j}$, and there exists an $i$ such that $u_{i}$ and $w_{i}$ are linearly dependent, but for each $j \neq i, u_{j}$ and $w_{j}$ are linearly independent. It follows from our computation above that the value of $i$ is uniquely determined by $z$; and we write $i=i(z)$. For $i=1, \ldots, t$, let $\Delta(i)=\{z \in \Delta \mid i(z)=i\}$. Then the subsets $\Delta(i)(1 \leq i \leq t)$ form a system of imprimitivity for $H$ in $\Delta$, and since $H$ is primitive on $\Delta$, it follows that they have size 1 . Thus $\Delta(1)=\{v\}$, and it follows that $H_{v}=H_{1}$. This means that $|\Delta|=t$. However, since $H$ induces an irreducible linear group on $\Omega, \Delta$ must contain a basis of $\Omega$ whence $|\Delta| \geq m^{t}>t$, which is a contradiction.

Lemma 3.11. Case (iii) above, with $t=2$, does not hold.

Proof. Suppose that (iii) holds, with $t=2$, so by Lemma 3.9, we may assume that $H \leq\left(N_{\mathrm{GL}(U)}(K) \circ N_{\mathrm{GL}(U)}(K)\right) . S_{2}$ and $H \cap(\mathrm{GL}(U) \circ \mathrm{GL}(U)) \leq K \circ K$, where $N_{\mathrm{GL}(U)}(K)$ is an irreducible subgroup of $\mathrm{GL}(U)$. Moreover, $L:=H \cap(K \circ K)$ has index 2 in $H$. As in Lemma 3.9 we will work in the group $N_{\mathrm{GL}(U)}(K)$ ? $S_{2}$, acting unfaithfully on $\Omega$. Let $\pi\left(x, x^{\prime}\right)$ and $\pi\left(y, y^{\prime}\right)$ be elements of $H \backslash L$, where $x, x^{\prime}, y, y^{\prime} \in N_{\mathrm{GL}(U)}(K)$ and $\pi=(12) \in S_{2}$. Then $L$ contains the elements

$$
\pi\left(x, x^{\prime}\right) \pi\left(y, y^{\prime}\right)=\left(x^{\prime} y, x y^{\prime}\right) \text { and } \pi\left(x, x^{\prime}\right)\left(y^{-1}, y^{\prime-1}\right) \pi=\left(x^{\prime} y^{-1}, x y^{-1}\right) .
$$

Hence $x \equiv y \equiv x^{\prime-1} \equiv y^{\prime-1}(\bmod K)$. Thus $H \leq N<S_{2}$, where $N=\langle K, x\rangle$, and $H$ contains $\pi\left(x, x^{-1} k\right)$ for some $k \in K$.

Let $\left\{e_{1}, \ldots, e_{m}\right\}$ be a basis for $U$, so that the set $\mathbb{B}$ of $m^{2}$ vectors $e_{i} \otimes e_{j}$ (for $i, j \leq m$ ) forms a basis for $\Omega$. Suppose first that $m=2$, so $n=4$, and since $H$ is insoluble, $2^{a} \geq 4$. Since $H$ is not realizable over a proper subfield, and $H$ is insoluble, it follows (see [3, Section 260]) that $H$ involves SL $\left(2,2^{a}\right)$. This implies that $H$ has an element of order $2^{a}-1$, contradicting Lemma 3.1.

Hence $m \geq 3$. Let $v=e_{1} \otimes e_{1}+e_{2} \otimes e_{2}$, and let $\operatorname{stab}(W)$ denote the subgroup of $\mathrm{GL}(U)$ which leaves the subspace $W:=\left\langle e_{1}, e_{2}\right\rangle$ invariant (setwise). We claim that $H_{v} \leq \operatorname{stab}(W) \gtrless S_{2}$. Let $\pi\left(h, h^{\prime}\right) \in H_{v}$. Then $v=v\left(h, h^{\prime}\right)=\left(e_{1} h\right) \otimes$ $\left(e_{1} h^{\prime}\right)+\left(e_{2} h\right) \otimes\left(e_{2} h^{\prime}\right)$. Set $e_{i} h=\sum_{l} c_{i l} e_{l}$ and $e_{i} h^{\prime}=\sum_{l} c_{i l}^{\prime} e_{l}$. Then $v=$ 
$\sum_{i, j}\left(c_{1 i} c_{1 j}^{\prime}+c_{2 i} c_{2 j}^{\prime}\right)\left(e_{i} \otimes e_{j}\right)$, so

$$
\begin{aligned}
& c_{11} c_{11}^{\prime}+c_{21} c_{21}^{\prime}=1 \\
& c_{12} c_{12}^{\prime}+c_{22} c_{22}^{\prime}=1, \quad \text { and } \\
& c_{1 i} c_{1 j}^{\prime}+c_{2 i} c_{2 j}^{\prime}=0, \quad \text { if }(i, j) \neq(1,1) \text { or }(2,2) .
\end{aligned}
$$

Define

$$
A_{i j}=\left(\begin{array}{ll}
c_{1 i} & c_{2 i} \\
c_{1 j} & c_{2 j}
\end{array}\right) \quad \text { and } \quad A_{i j}^{\prime}=\left(\begin{array}{cc}
c_{1 i}^{\prime} & c_{2 i}^{\prime} \\
c_{1 j}^{\prime} & c_{2 j}^{\prime}
\end{array}\right)
$$

The equations above imply that $A_{12}\left(A_{12}^{\prime}\right)^{\mathrm{tr}}=I$, and for $j \geq 3$,

$$
A_{12}\left(A_{1 j}^{\prime}\right)^{\mathrm{tr}}=\left(\begin{array}{ll}
1 & 0 \\
0 & 0
\end{array}\right)
$$

and hence

$$
\left(A_{1 j}^{\prime}\right)^{\operatorname{tr}}=A_{12}^{-1}\left(\begin{array}{ll}
1 & 0 \\
0 & 0
\end{array}\right)=\left(\begin{array}{ll}
b & 0 \\
c & 0
\end{array}\right)
$$

for some $b, c$. It follows that $c_{1 j}^{\prime}=c_{2 j}^{\prime}=0$ for all $j \geq 3$. Similarly, for $j \geq 3$,

$$
A_{12}^{\prime} A_{1 j}^{\mathrm{tr}}=\left(\begin{array}{ll}
1 & 0 \\
0 & 0
\end{array}\right)
$$

and hence

$$
A_{1 j}^{\mathrm{tr}}=\left(A_{12}^{\prime}\right)^{-1}\left(\begin{array}{ll}
1 & 0 \\
0 & 0
\end{array}\right)=\left(\begin{array}{ll}
b^{\prime} & 0 \\
c^{\prime} & 0
\end{array}\right)
$$

for some $b^{\prime}, c^{\prime}$, so $c_{1 j}=c_{2 j}=0$ for all $j \geq 3$. Thus $h, h^{\prime}$ both leave $W=\left\langle e_{1}, e_{2}\right\rangle$ invariant, and so $H_{v} \leq \operatorname{stab}(W) \gtrless S_{2}$, proving our claim.

Since $m \geq 3, H \cap\left(\operatorname{stab}(W) \geq S_{2}\right)$ is a proper subgroup of $H$ containing $H_{v}$, and since $H_{v}$ is maximal in $H$ it follows that $H_{v}=H \cap\left(\operatorname{stab}(W) \geq S_{2}\right)$. The same argument shows that $H_{v\left(u, u^{\prime}\right)}=H \cap\left(\operatorname{stab}(W) 2 S_{2}\right)$, where $v\left(u, u^{\prime}\right)=u \otimes u+u^{\prime} \otimes u^{\prime}$, for any basis $u, u^{\prime}$ of $W$. Suppose that $H_{v}$ stabilises $\left\langle e_{1} \otimes e_{1}\right\rangle$. By maximality, $H_{v}$ is equal to the stabilizer of $\left\langle e_{1} \otimes e_{1}\right\rangle$, and by Lemma 3.1, we have that $H_{v}$ stabilises $e_{1} \otimes e_{1}$. The same argument proves that $H_{v}$ stabilises $u \otimes u$ for each $u \in W$, and applying it again to any 2-dimensional subspace of $U$ containing $e_{1}$ we have that $H_{v}$ stabilises $u \otimes u$ for each $u \in U$. It follows that $H_{v} \cap(\operatorname{GL}(U) \circ \operatorname{GL}(U))$ is a subgroup of scalar matrices, and by Lemma 3.1 is trivial. Hence $\left|H_{v}\right| \leq 2$. Since $H$ is primitive on the orbit $v^{H}$, it follows (see $[30,18.7]$ ) that $H$ is soluble, which is a contradiction. Thus $H_{v}$ does not stabilise $\left\langle e_{1} \otimes e_{1}\right\rangle$.

If $a>1$ then, taking $b \in \mathrm{GF}\left(2^{a}\right) \backslash\{0,1\}$ and $u=e_{1}, u^{\prime}=b e_{2}$, we see that $H_{v}$ stabilises $v^{\prime}=e_{1} \otimes e_{1}+b^{2} e_{2} \otimes e_{2}$, and hence also stabilises $\left\langle e_{1} \otimes e_{1}\right\rangle$, which is a contradiction. Hence $\mathrm{GF}\left(2^{a}\right)=\mathrm{GF}(2)$. In this case the condition that $H_{v}$ stabilise $v\left(u, u^{\prime}\right)$, for all bases $u, u^{\prime}$ for $W$, implies that the subgroup of $\mathrm{GL}(W) \geq S_{2}$ induced 
by $H_{v}$ is contained in $\left\langle\left(h, h^{-1}\right)\right\rangle \cdot\langle\pi\rangle \cong S_{3}$, where $\pi: u \otimes v \mapsto v \otimes u$ for all $u, v$, and where

$$
h=\left(\begin{array}{ll}
0 & 1 \\
1 & 1
\end{array}\right)
$$

with respect to the basis $e_{1}, e_{2}$ of $W$. This means also that the subgroup of GL( $\left.W\right) 2 S_{2}$ induced by $L \cap H_{v}$ is contained in $\left\langle\left(h, h^{-1}\right)\right\rangle \cong Z_{3}$. Note that $h: e_{1} \rightarrow e_{2} \rightarrow e_{1}+e_{2}$.

Recall that $H \leq N<S_{2}$, where $N=\langle K, x\rangle \leq N(K)$, and $K$ is the subgroup of $\mathrm{GL}(U)$ induced by $H \cap(\mathrm{GL}(U) \times \mathrm{GL}(U))$. Also, $H$ contains $\pi\left(x, x^{-1} k\right)$ for some $k \in K$. Suppose that the group $N$ is intransitive on the nonzero vectors of $U$. Then we may assume that $e_{1}, e_{2}$ lie in different $N$-orbits. In this case the subgroup of GL $(W) \gtrless S_{2}$ induced by $H_{v}$ is contained in $\langle\pi\rangle$. In particular $H_{v}$ stabilises $e_{1} \otimes e_{1}$ which is not the case. Hence $N$ is transitive on $U^{\#}$. By the definition of $a$, and since $a=1$, we know that $N$ is not contained in $\operatorname{GL}\left(m / a^{\prime}, 2^{a^{\prime}}\right) \cdot a^{\prime}$ for any divisor $a^{\prime}>1$ of $m$. Hence, by [17, Appendix], $N$ is either $\operatorname{GL}(m, 2)$, or $\operatorname{Sp}(m, 2)$ ( $m$ even), or $N=\mathrm{G}_{2}(2)^{\prime}$ or $\mathrm{G}_{2}(2)$ (with $m=6$ ), or $N=A_{7}$ (with $m=4$ ). We obtain a final contradiction by showing that in all cases there is a 2-dimensional subspace $W$ of $U$ for which the stabilizer of $W \otimes W$ in $L$ induces at least an $S_{3}$ on $W \otimes W$, contradicting the fact that this stabilizer was shown to induce at most $Z_{3}$. This holds for any 2subspace $W$ if $N=\operatorname{GL}(m, 2), A_{7}$, or $G_{2}(2)$, for any non-singular 2-subspace if $N=\mathrm{Sp}(m, 2)$, and for 315 of the 6512 -subspaces if $N=G_{2}(2)^{\prime}$ (the information about $G_{2}(2)$ and $G_{2}(2)^{\prime}$ was checked using GAP).

\section{Simply primitive affine groups}

In this section we prove Theorem 1.3 and give further information (see Theorem 4.8) about the potential simply primitive, extremely primitive groups that may not have been listed in the statement of Theorem 1.3. For the entire section, let $G \leq S_{2 d}$ be a simply primitive permutation group of affine type, of structure $G=Z_{2}^{\bar{d}} . H$ with $H$ almost simple. Let $\mathcal{M}$ denote the set of maximal subgroups of $H$, considered as matrix groups acting on $V=\mathrm{GF}(2)^{d}$. For $M \in \mathcal{M}$, let fix $(M)$ denote the set of fixed vectors of $M$.

One of our major tools is the following simple observation.

\section{Lemma 4.1.}

$$
\sum_{M \in \mathcal{M}}(|\operatorname{fix}(M)|-1) \leq 2^{d}-1,
$$

with equality if and only if $G$ is extremely primitive. In particular, if $|\mathcal{M}|<2^{d / 2}$, then $G$ is not extremely primitive.

Proof. For distinct $M_{1}, M_{2} \in \mathcal{M}$, we have fix $\left(M_{1}\right) \cap \operatorname{fix}\left(M_{2}\right)=\{0\}$ because there is no nontrivial fixed vector of $\left\langle M_{1}, M_{2}\right\rangle=H$. Hence each nonzero $v \in V$ occurs 
in at most one set fix $(M)$ and $\sum_{M \in \mathcal{M}}(|\operatorname{fix}(M)|-1) \leq 2^{d}-1$. Now $G$ is extremely primitive if and only if each nonzero $v \in V$ indeed occurs in fix $(M)$ for some $M \in \mathcal{M}$, that is, equality holds.

Now, for each $M \in \mathcal{M}, \operatorname{dim}(\operatorname{fix}(M)) \leq d / 2$, since otherwise fix $(M)$ and fix $\left(M^{g}\right)$ would have nontrivial intersection for $g \in H \backslash M$. Thus if $|\mathcal{M}|<2^{d / 2}$, then $\sum_{M \in \mathcal{M}}(|\operatorname{fix}(M)|-1)<2^{d}-1$ and hence $G$ is not extremely primitive by the previous paragraph.

Lemma 4.2. The groups $Z_{2}^{d}$.H listed in the statement of Theorem 1.3 are extremely primitive.

Proof. For the groups $G$ in Theorem 1.3 (a) with $d \leq 22$, and those in parts (b) and (c) with $d \leq 12$, either we constructed $G$ using the primitive group library of GAP, or we constructed $H$ as a matrix group using the at lasrep package of GAP [31], and then computed the $H$-orbits in $V=\mathrm{GF}(2)^{d}$ and the permutation actions of $H$ on these orbits. We verified that each of these groups is extremely primitive. For the group $G=Z_{2}^{24} . C o_{1}$, generators of maximal subgroups $M$ of type $C o_{2}, Z_{2}^{11} \cdot M_{24}$, and $\mathrm{Co}_{3}$ were constructed using at lasrep, enabling us to compute the dimensions of their fixed point spaces. Then we checked that the sum of $|\operatorname{fix}(M)|-1$ over the maximal subgroups $M$ of $H$ isomorphic to any of these three subgroups adds up to $2^{24}-1$. Hence, by Lemma 4.1, this group $G$ is also extremely primitive.

It is well known that the groups $H=\Omega^{ \pm}(2 k, 2)$ and $\Omega^{ \pm}(2 k, 2) .2$, where $k \geq 7$, have two orbits on GF(2) ${ }^{2 k} \backslash\{0\}$ (the sets of singular and nonsingular vectors) and $H$ acts primitively on each of these orbits.

What remains to prove is that the groups in Theorem 1.3 (b) with $d \geq 14$ are extremely primitive. In these cases $H=A_{n}$ or $S_{n}$ with $n \in\{2 k+1,2 k+2\}$, and $H$ acts on $V=\mathrm{GF}(2)^{2 k}$ as on the deleted permutation module for its natural action of degree $n$. Let $U=\operatorname{GF}(2)^{n}$ be the $n$-dimensional permutation module for the natural action of $H$, and let $W=\left\{\left(u_{1}, \ldots, u_{n}\right) \in U \mid \sum_{i=1}^{n} u_{i}=0\right\}$. The stabilizer in $H$ of a vector in $U$ with $\ell$ nonzero coordinates is $H \cap\left(S_{\ell} \times S_{n-\ell}\right)$, and two vectors of $U$ are in the same $H$-orbit if and only if they have the same number of nonzero coordinates. Thus, $H$ acts primitively on each of its orbits in $U$, apart from the orbit of vectors with $k+1$ nonzero entries when $n=2 k+2$. The subspace $W$ is the union of those $H$-orbits that contain vectors with an even number of nonzero coordinates, so $H$ acts primitively on each of its orbits in $W$ except the orbit of vectors with $k+1$ nonzero entries in the case $n=2 k+2, k$ odd. If $n=2 k+1$ then $V=W$ and so $G$ is extremely primitive. If $n=2 k+2$ then $V$ is the factor space of $W$, obtained by identifying the pairs of vectors $v_{1}=\left(u_{1}, \ldots, u_{n}\right)$ and $v_{2}=(1,1, \ldots, 1)-\left(u_{1}, \ldots, u_{n}\right)$, for all $v_{1} \in W$. Suppose without loss of generality that $v_{1}$ has $\ell$ nonzero entries and $\ell \leq n / 2$. Note that $\ell$ is even since $v_{1} \in W$. If $\ell<n / 2$ then the action of $H$ on the orbit $\Delta$ of the pair $\left\{v_{1}, v_{2}\right\} \in V$ is permutationally isomorphic to the primitive action of $H$ on the orbit of $v_{1} \in W$, while if $\ell=n / 2$ then $v_{1}, v_{2}$ correspond to disjoint 
$\ell$-sets, and so the $H$-action on $\Delta$ is permutationally isomorphic to its primitive action on partitions with two parts of size $n / 2$. Hence in all cases $H$ acts primitively on all of its orbits and $G$ is extremely primitive.

Now we start investigating the Lie-type simple groups of characteristic 2 that may occur as $\operatorname{Soc}(H)$ for an extremely primitive group $G$.

For a field $F$ of characteristic 2 and an $F$-vectorspace $V_{0}$, the Frobenius automorphism $\sigma: x \mapsto x^{2}$ of $F$ induces a semilinear map $\sigma: \sum_{i} \beta_{i} b_{i} \mapsto \sum_{i} \beta_{i}^{2} b_{i}$ of $V_{0}$ with respect to a given $F$-basis $B=\left\{b_{1}, \ldots, b_{m}\right\}$ of $V_{0}$. If $V_{0}$ is an $F H$-module and $k$ is a positive integer, then by $V_{0}^{(k)}$ we mean the 'twisted' $F H$-module with underlying vector space $V_{0}$ such that for $h \in H, h: v \mapsto v \sigma^{-k} h \sigma^{k}$. For $h=\left(h_{i j}\right) \in \operatorname{GL}(m, F)$, where the matrix is relative to the basis $B$, the Frobenius automorphism $\sigma$ satisfies $\sigma^{-1} h \sigma=\left(h_{i j}^{\sigma}\right)$ in $\Gamma \mathrm{L}(m, F)$, and we write $h^{\sigma}=\left(h_{i j}^{\sigma}\right)$. Thus if $h \in H$ acts on $V_{0}$ with matrix $\left(h_{i j}\right) \in \mathrm{GL}(m, F)$ relative to the basis $B$, then $h$ acts on $V_{0}^{(k)}$ by $h: v \mapsto v h^{\sigma^{k}}$. Moreover, the tensor product $W=V_{0} \otimes V_{0}^{(1)} \otimes \cdots \otimes V_{0}^{(e-1)}$ becomes an $F H$-module with action defined by $h: v_{1} \otimes v_{2} \otimes \cdots \otimes v_{e} \mapsto v_{1} h \otimes v_{2} h^{\sigma} \otimes \cdots \otimes v_{e} h^{\sigma^{e-1}}$. For each $e$-tuple $\boldsymbol{i}=\left(i_{1}, \ldots, i_{e}\right)$, with $1 \leq i_{j} \leq m$ for each $j$, let

$$
\tilde{b}_{\boldsymbol{i}}=b_{i_{1}} \otimes b_{i_{2}} \otimes \cdots \otimes b_{i_{e}}
$$

so that the set $\mathbb{B}$ of all such $\tilde{b}_{\boldsymbol{i}}$ is an $F$-basis for $W$. If $\pi$ denotes the element of $\operatorname{GL}(W)$ defined by $\tilde{b}_{\boldsymbol{i}} \pi=\tilde{b}_{\boldsymbol{i}^{*}}$, where $\boldsymbol{i}^{*}=\left(i_{e}, i_{1}, \ldots, i_{e-1}\right)$, then $\tilde{b}_{\boldsymbol{i}} h^{\sigma}=\tilde{b}_{\boldsymbol{i}} \pi h \pi^{-1}$, for each $\tilde{b}_{\boldsymbol{i}} \in \mathbb{B}$. Thus the element $\sigma$ of $\Gamma \mathrm{L}(m, F)$ acts on $W$ as $\pi^{-1}$, and we shall denote it still as $\sigma$.

Lemma 4.3. Suppose that $G=Z_{2}^{d} \cdot H$ is extremely primitive and simply primitive, that $H$ is of Lie type defined over the field $\mathrm{GF}\left(2^{e}\right)$ with socle $H_{0}$, and let a be the largest integer such that $H \leq \mathrm{GL}\left(d / a, 2^{a}\right) . a$. Then the following hold.

(i) $a=1$ and $d=m^{e}$ for some integer $m$;

(ii) there is an absolutely irreducible $m$-dimensional $\mathrm{GF}\left(2^{e}\right) H_{0}$-module $V_{0}$ with basis $B$ such that $V \otimes \mathrm{GF}\left(2^{e}\right)=V_{0} \otimes V_{0}^{(1)} \otimes \cdots \otimes V_{0}^{(e-1)}$ with basis $\mathbb{B}$ defined as above. Moreover, $V$ can be identified with the $\mathrm{GF}(2)$-subspace $\tilde{V}$ consisting of all vectors $\sum_{\boldsymbol{i}} \lambda_{\boldsymbol{i}} \tilde{b}_{\boldsymbol{i}}$ such that $\lambda_{\boldsymbol{i}^{*}}=\lambda_{\boldsymbol{i}}^{2}$ for all $\tilde{b}_{\boldsymbol{i}} \in \mathbb{B}$.

Proof. First note that $2^{e}-1$ divides $|H|$. Hence, if $\operatorname{gcd}\left(2^{e}-1,2^{a}-1\right)>1$ or, equivalently, $\operatorname{gcd}(e, a)>1$ then $H$ has an element of prime order $r \mid 2^{a}-1$. This contradicts Lemma 3.1, and hence $\operatorname{gcd}(e, a)=1$.

Let $H_{0}:=\operatorname{Soc}(H)$. By Lemma 3.6, $H_{0}$ is absolutely irreducible on $V$ regarded as a $\mathrm{GF}\left(2^{a}\right)$-module $\mathrm{GF}\left(2^{a}\right)^{d / a}$ and $H$ is not realisable over a proper subfield. Hence we are in the situation considered in $[13,5.4 .6,5.4 .7]$. If $H_{0}$ is untwisted or of type ${ }^{2} B_{2},{ }^{2} G_{2}$, or ${ }^{2} F_{4}$ then $[13,5.4 .6(\mathrm{i}), 5.4 .7(\mathrm{~b})]$ imply that $a \mid e$. Using $\operatorname{gcd}(a, e)=1$, we 
obtain $a=1$. If $H_{0}$ is of type ${ }^{2} A_{n},{ }^{2} D_{n}$, or ${ }^{2} E_{6}$ then [13, 5.4.6(ii)] implies that either $a \mid e$ whence $a=1$, or $a \mid 2 e$ but $a$ does not divide $e$. In the latter case, $\operatorname{gcd}(a, e)=1$ gives that $a=2$. However, $H_{0}$ has elements of order 3 , contradicting Lemma 3.1 so in this case also $a=1$. Finally, if $H_{0}$ is of type ${ }^{3} D_{4}$ then [13, 5.4.7(a)] implies that either $a \mid e$ whence $a=1$, or $a \mid 3 e$ but $a$ does not divide $e$. In the latter case $a=3$ and, since $H_{0}$ contains elements of order 7, Lemma 3.1 gives a contradiction. Hence in all cases $a=1$.

Appealing again to $[13,5.4 .6,5.4 .7]$, and to the proof of [16, Theorem 2.3] (referred to in $[13,5.4 .7(\mathrm{~b})]$ ), we obtain that there is an absolutely irreducible $\mathrm{FH}_{0^{-}}$ module $M$ of dimension $m$ over the algebraic closure $F$ of GF $\left(2^{e}\right)$, with basis $B=$ $\left\{b_{1}, \ldots, b_{m}\right\}$, such that $V \otimes F$ is isomorphic to the $F H_{0}$-module $W:=M \otimes M^{(1)} \otimes$ $\cdots \otimes M^{(e-1)}$ with basis $\mathbb{B}$, defined as above. In particular $d=m^{e}$, completing the proof of part (i). Moreover, $H \leq \Gamma \mathrm{L}(W)=\Gamma \mathrm{L}(d, F)$.

Since $a=1$, the GF(2) $H$-module $V$ can be identified with a $d$-dimensional, $H$ invariant, $\mathrm{GF}(2)$-subspace of $W$. Such a subspace must be fixed elementwise by $\sigma$ (under the action described above), and hence must be contained in the subset $\widetilde{V}$ of all vectors of $W$ fixed by $\sigma$. It is straightforward to compute that $\widetilde{V}$ consists of all $\sum_{\boldsymbol{i}} \lambda_{\boldsymbol{i}} \tilde{b}_{\boldsymbol{i}}$ such that $\lambda_{\boldsymbol{i}^{*}}=\lambda_{\boldsymbol{i}}^{2}$ for all $\boldsymbol{i}$. Clearly $\widetilde{V}$ is a GF(2)-subspace. Let $\sum_{\boldsymbol{i}} \lambda_{\boldsymbol{i}} \tilde{b}_{\boldsymbol{i}} \in \tilde{V}$. For any $\boldsymbol{i}$, there is a smallest positive integer $e(\boldsymbol{i})$ such that $i_{j}=i_{j+e(i)}$ for all $j$ (reading subscripts modulo $e$ ), and since $\lambda_{\boldsymbol{i}^{*}}=\lambda_{\boldsymbol{i}}^{2}$ for all $\boldsymbol{i}$, it follows that $\lambda_{\boldsymbol{i}}^{2^{e(i)}}=\lambda_{\boldsymbol{i}}$ so that $\lambda_{i} \in \operatorname{GF}\left(2^{e(i)}\right)$. Also, clearly $e(i)$ divides $e$, and hence $\lambda_{i} \in \operatorname{GF}\left(2^{e}\right)$. Further, cyclically shifting the subscripts $\boldsymbol{i}$ defines an equivalence on the set of basis vectors $\mathbb{B}$, where $\tilde{b}_{\boldsymbol{i}}$ and $\tilde{b}_{\boldsymbol{i}^{\prime}}$ are equivalent if $\boldsymbol{i}^{\prime}$ can be obtained from $\boldsymbol{i}$ by repeated cyclic shifts, and there are $e(\boldsymbol{i})$ basis vectors in the equivalence class of $\tilde{b}_{\boldsymbol{i}}$. Moreover, since $\lambda_{\boldsymbol{i}^{*}}=\lambda_{\boldsymbol{i}}^{2}$, the coefficient $\lambda_{\boldsymbol{i}}$ (which can be chosen arbitrarily in $\operatorname{GF}\left(2^{e(i)}\right)$ ) uniquely determines the coefficients $\lambda_{\boldsymbol{i}^{\prime}}$ for all other $\tilde{b}_{\boldsymbol{i}^{\prime}}$ equivalent to $\tilde{b}_{\boldsymbol{i}}$. It follows that $|\tilde{V}|$ is equal to the product of $2^{e(i)}$ over all equivalence classes, that is, $|\tilde{V}|=2^{m^{e}}=2^{d}$ so $\widetilde{V}$ has $\operatorname{GF}(2)$-dimension $d$. Since also $V$ has $\operatorname{GF}(2)$-dimension $d$, it follows that $V$ can be identified with $\widetilde{V}$, and in particular that $\widetilde{V}$ is $H$-invariant.

Recall that $\sigma$ acts trivially on $\widetilde{V}$. We now examine the action on $\widetilde{V}$ of an arbitrary $h \in H \cap \mathrm{GL}(m, F)$ with matrix $\left(h_{i j}\right)$ relative to $B$. For each $\boldsymbol{i}=\left(i_{1}, \ldots, i_{e}\right)$,

$$
\begin{aligned}
\tilde{b}_{\boldsymbol{i}} h & =b_{i_{1}} h \otimes b_{i_{2}} h^{\sigma} \otimes \cdots \otimes b_{i_{e}} h^{\sigma^{e-1}} \\
& =\otimes_{k=1}^{e}\left(\sum_{j_{k}}\left(h_{i_{k} j_{k}} b_{j_{k}}\right)^{\sigma^{k-1}}\right)=\otimes_{k=1}^{e}\left(\sum_{j_{k}}\left(h_{i_{k} j_{k}}^{2^{k-1}} b_{j_{k}}\right)\right) .
\end{aligned}
$$

Consider the tuples $\boldsymbol{i}=(i, i \ldots, i)$ and $\boldsymbol{i}^{\prime}=(j, j \ldots, j)$, and note that $e(\boldsymbol{i})=$ $e\left(\boldsymbol{i}^{\prime}\right)=1$. Then $\tilde{b}_{\boldsymbol{i}} \in \tilde{V}$ and hence $\tilde{b}_{\boldsymbol{i}} h \in \widetilde{V}$. Thus the coefficient of $\tilde{b}_{\boldsymbol{i}^{\prime}}$ in $\tilde{b}_{\boldsymbol{i}} h$, which by the above calculation is $\prod_{k=1}^{e} h_{i j}^{2^{k-1}}=h_{i j}^{2^{e}-1}$, should lie in $\operatorname{GF}\left(2^{e\left(i^{\prime}\right)}\right)=\operatorname{GF}(2)$. Hence $h_{i j}^{2^{e}-1}=0$ or 1 , or equivalently, $h_{i j} \in \mathrm{GF}\left(2^{e}\right)$. Since this holds for all $i, j$, it follows that the matrix $\left(h_{i j}\right) \in \operatorname{GL}\left(m, 2^{e}\right)$. Thus $\operatorname{GF}\left(2^{e}\right)$ is a splitting field 
for this representation of $H$ and hence $\tilde{V}$ lies in the $\operatorname{GF}\left(2^{e}\right) H$-invariant subspace $V_{0} \otimes V_{0}^{(1)} \otimes \cdots \otimes V_{0}^{(e-1)}$, where $V_{0}$ is the restriction of $M$ to $\mathrm{GF}\left(2^{e}\right)$. This completes the proof.

Lemma 4.4. Let $G, H, e, V_{0}, \mathbb{B}, \tilde{V}$ be as in Lemma 4.3 with $G$ extremely primitive. Then either $e=m=2$ and $\operatorname{Soc}(H)=\operatorname{SL}(2,4)$ as in Theorem $1.3(\mathrm{~b})$, or $e=1$.

Proof. Suppose that $e \geq 2$. For $v \in \tilde{V}$ and $\tilde{b} \in \mathbb{B}$, let $c_{v}(\tilde{b})$ denote the coefficient of $\tilde{b}$ in the expression for $v$ as a linear combination of the basis $\mathbb{B}$, that is, $v=$ $\sum_{\tilde{b} \in \mathbb{B}} c_{v}(\tilde{b}) \tilde{b}$.

For $1 \leq j \leq m$, let $M_{j}$ denote the stabilizer in $H$ of the vector $v_{j}:=\tilde{b}_{(j, \ldots, j)}=$ $b_{j} \otimes \cdots \otimes b_{j} \in \mathbb{B}$. Since $G$ is extremely primitive, $M_{j}$ is maximal in $H$, so $M_{j}=H_{\left\langle b_{j}\right\rangle}$ in the $H$-action on $V_{0}$. In particular the $M_{j}$ are non-trivial, and hence are not all equal. Thus there are indices $j_{1}, j_{2}$ such that $M_{j_{1}} \neq M_{j_{2}}$. Without loss of generality, we may assume that $j_{1}=1$ and $j_{2}=2$.

Next, consider the stabilizer $M_{1,2}$ of $v:=v_{1}+v_{2} \in \tilde{V}$. Since $H$ is extremely primitive, $M_{1,2}$ is maximal in $H$, and therefore contains $M_{1} \cap M_{2}$ as a proper subgroup. Suppose that $h \in \operatorname{GL}\left(m, 2^{e}\right)$ fixes $v$, and in the action of $h$ on $V_{0}$ let

$$
b_{1} h=\sum_{j=1}^{m} \alpha_{1 j} b_{j}, \quad b_{2} h=\sum_{j=1}^{m} \alpha_{2 j} b_{j}
$$

with $\alpha_{i, j} \in \mathrm{GF}\left(2^{e}\right)$. Since $v h=v \in \tilde{V}$, we have $c_{v h}\left(\tilde{b}_{\boldsymbol{i}} *\right)=c_{v h}\left(\tilde{b_{i}}\right)^{2}$, for all $\boldsymbol{i}$, by Lemma 4.3. For each $j$,

$$
c_{v h}\left(v_{j}\right)=\prod_{i=0}^{e-1} \alpha_{1 j}^{2^{i}}+\prod_{i=0}^{e-1} \alpha_{2 j}^{2^{i}}=\alpha_{1 j}^{2^{e}-1}+\alpha_{2 j}^{2^{e}-1}
$$

and each summand lies in $\operatorname{GF}(2)=\{0,1\}$, so $c_{v h}\left(v_{j}\right)=1$ if and only if exactly one of $\alpha_{1 j}, \alpha_{2, j}$ is nonzero. In particular, exactly one of $\alpha_{11}$ and $\alpha_{21}$ is nonzero. If, for some $j \geq 3$, both $\alpha_{1 j}, \alpha_{2, j}$ are nonzero, then

$$
c_{v h}\left(\tilde{b}_{(1, j, \ldots, j)}\right)=\alpha_{11} \alpha_{1 j}^{2+4+\cdots+2^{e-1}}+\alpha_{21} \alpha_{2 j}^{2+4+\cdots+2^{e-1}}
$$

is nonzero because exactly one of the summands is nonzero. This contradicts the fact that $v h=v$. Thus, for each $j \geq 3, \alpha_{1 j}=\alpha_{2 j}=0$, and so either $b_{1} h=$ $\alpha_{11} b_{1}, b_{2} h=\alpha_{22} b_{2}$, or $b_{1} h=\alpha_{12} b_{2}, b_{2} h=\alpha_{21} b_{1}$. Moreover each such element $h$ fixes $v$. Elements $h$ in $H$ of the first type lie in the proper subgroup $M_{1} \cap M_{2}$ of $M_{1,2}$, and hence, in its action on $V_{0}, M_{1,2}$ fixes $\left\langle b_{1}, b_{2}\right\rangle$ and interchanges the 1-spaces $\left\langle b_{1}\right\rangle$ and $\left\langle b_{2}\right\rangle$. Since $M_{j}=H_{\left\langle b_{j}\right\rangle}$ for each $j$, it follows that $\left|M_{1,2}: M_{1} \cap M_{2}\right|=2$. If $m \geq 3$ then $M_{1,2}$ lies in the proper subgroup $H_{\left\langle b_{1}, b_{2}\right\rangle}$, and the maximality of $M_{1,2}$ 
implies that $M_{1,2}=H_{\left\langle b_{1}, b_{2}\right\rangle}$. On the other hand, if $m=2$, we only know that $M_{1,2}$ is the setwise stabilizer of $\left\{\left\langle b_{1}\right\rangle,\left\langle b_{2}\right\rangle\right\}$ and maximal in $H$.

Next suppose that $e \geq 3$ and consider the stabilizers $M=M(\lambda)$ in $H$ of the vectors of the form

$$
u=u(\lambda):=\lambda \tilde{b}_{(1,2, \ldots, 2)}+\lambda^{2} \tilde{b}_{(2,1,2, \ldots, 2)}+\cdots+\lambda^{2^{e-1}} \tilde{b}_{(2, \ldots, 2,1)} \in \tilde{V}
$$

where $\lambda \in \operatorname{GF}\left(2^{e}\right)^{\#}$. Suppose that $h \in \mathrm{GL}\left(m, 2^{e}\right)$ such that $h$ fixes $u$, and suppose that (1) holds. Since $u h=u$, for each $j$,

$$
c_{u h}\left(v_{j}\right)=\sum_{i=0}^{e-1} \lambda^{2^{i}} \alpha_{1 j}^{2^{i}} \alpha_{2 j}^{2^{e}-1-2^{i}}=0,
$$

so if $\alpha_{2 j} \neq 0$ then

$$
\sum_{i=0}^{e-1}\left(\lambda \alpha_{1 j} \alpha_{2 j}^{-1}\right)^{2^{i}}=0
$$

Now

$$
c_{u h}\left(\tilde{b}_{(1,2, \ldots, 2)}\right)=\lambda \alpha_{11} \alpha_{22}^{2^{e}-2}+\alpha_{21} \sum_{i=1}^{e-1} \lambda^{2^{i}} \alpha_{12}^{2^{i}} \alpha_{22}^{2^{e}-2-2^{i}}=\lambda
$$

and since each summand is a multiple of $\alpha_{22}$, it follows that $\alpha_{22} \neq 0$. (This deduction requires $e \geq 3$.) Thus $\alpha_{22}^{2^{e}-1}=1$, and using (2) we obtain

$$
\alpha_{11} \alpha_{22}^{-1}+\alpha_{21} \alpha_{22}^{-1}\left(\alpha_{12} \alpha_{22}^{-1}\right)=1 \text {, so } \alpha_{22}^{2}=\alpha_{11} \alpha_{22}+\alpha_{21} \alpha_{12} \text {. }
$$

Next,

$$
c_{u h}\left(\tilde{b}_{(2,1, \ldots, 1)}\right)=\lambda \alpha_{12} \alpha_{21}^{2^{e}-2}+\alpha_{22} \sum_{i=1}^{e-1} \lambda^{2^{i}} \alpha_{11}^{2^{i}} \alpha_{21}^{2^{e}-2-2^{i}}=0 .
$$

Suppose that $\alpha_{21} \neq 0$. Then this equation becomes, using (2), $\alpha_{12}=\alpha_{22} \alpha_{11} \alpha_{21}^{-1}$. Substituting in (3) yields $\alpha_{22}=0$, which is a contradiction. Thus $\alpha_{21}=0$, and (3) implies $\alpha_{11}=\alpha_{22}$. Using these values we find, for $j \geq 3$,

$$
c_{u h}\left(\tilde{b}_{(1, j, 2, \ldots, 2)}\right)=\lambda \alpha_{11} \alpha_{2 j}^{2} \alpha_{22}^{2^{e}-4}=0
$$

which implies $\alpha_{2 j}=0$ for all $j \geq 3$. Then we have also

$$
c_{u h}\left(\tilde{b}_{(j, 2, \ldots, 2)}\right)=\lambda \alpha_{1 j} \alpha_{22}^{2^{e}-2}=0
$$

which implies that $\alpha_{1 j}=0$ for all $j \geq 3$. Thus $h$ fixes $\left\langle b_{1}, b_{2}\right\rangle$ setwise. If $m \geq 3$, then this implies that $M \leq M_{1,2}=H_{\left\langle b_{1}, b_{2}\right\rangle}$, and by the maximality of $M$, equality holds. This in turn implies that some element of $M$ interchanges $\left\langle b_{1}\right\rangle$ and $\left\langle b_{2}\right\rangle$, but 
we have just proved that this is not the case. Thus $m=2$. Now the fact that $\alpha_{21}=0$ implies that $h$ fixes $\left\langle b_{2}\right\rangle$. Hence $M \leq M_{2}=H_{\left\langle b_{2}\right\rangle}$ and maximality implies that $M=M_{2}$. In fact $M_{2}=M(\lambda)$ for each $\lambda \in \mathrm{GF}\left(2^{e}\right)^{\#}$. Since $M_{2} \neq M_{1}$, some element of $M_{2}$ moves $\left\langle b_{1}\right\rangle$, and we may assume that $h$ is such an element. Thus $\alpha_{12} \neq 0$, and (2) with $j=2$ implies that

$$
\sum_{i=0}^{e-1}(\lambda \alpha)^{2^{i}}=0 \quad \text { where } \alpha=\alpha_{21} \alpha_{22}^{-1}
$$

that is to say, $\lambda \alpha$ has trace 0 in $\mathrm{GF}\left(2^{e}\right)$ (over $\mathrm{GF}(2)$ ). Since $\alpha \neq 0$, and this holds for all $\lambda \neq 0$, it follows that all nonzero elements of $\mathrm{GF}\left(2^{e}\right)$ have trace zero, which is a contradiction. Thus we have proved that $e=2$.

If $e=m=2$, then $\operatorname{Soc}(H)=\operatorname{SL}(2,4)<\operatorname{GL}(4,2)$ and we have the unique degree 4 absolutely irreducible representation of this group, that is, Theorem 1.3 (b) holds. Assume then that $m \geq 3$, so $M_{1,2}=H_{\left\langle b_{1}, b_{2}\right\rangle}$ and elements of this subgroup fix or interchange $\left\langle b_{1}\right\rangle$ and $\left\langle b_{2}\right\rangle$. To complete the proof we consider the vectors $w=w(\lambda):=\lambda \tilde{b}_{(1,2)}+\lambda^{2} \tilde{b}_{(2,1)}$, where $\lambda \in \mathrm{GF}(4)^{\#}$. Note that $\lambda^{3}=1$. It follows from Lemma 4.3 that $w \in \tilde{V}$. Suppose that $h \in \operatorname{GL}\left(V_{0}\right)=\operatorname{GL}(m, 4)$ such that $h$ fixes $w$ and suppose that (1) holds. Then, for each $j$,

$$
c_{w h}\left(v_{j}\right)=\lambda \alpha_{1 j} \alpha_{2 j}^{2}+\lambda^{2} \alpha_{2 j} \alpha_{1 j}^{2}=0, \text { so } \alpha_{1 j} \alpha_{2 j}^{2}=\lambda \alpha_{2 j} \alpha_{1 j}^{2} \text {. }
$$

Next we compute, where $j \geq 3$,

$$
\begin{aligned}
& c_{w h}\left(\tilde{b}_{(1,2)}\right)=\lambda \quad \Longrightarrow \quad \lambda \alpha_{11} \alpha_{22}^{2}+\lambda^{2} \alpha_{21} \alpha_{12}^{2}=\lambda, \\
& c_{w h}\left(\tilde{b}_{(2,1)}\right)=\lambda^{2} \quad \Longrightarrow \quad \lambda \alpha_{12} \alpha_{21}^{2}+\lambda^{2} \alpha_{22} \alpha_{11}^{2}=\lambda^{2}, \\
& c_{w h}\left(\tilde{b}_{(j, 1)}\right)=0 \quad \Longrightarrow \quad \alpha_{1 j} \alpha_{21}^{2}=\lambda \alpha_{2 j} \alpha_{11}^{2}, \\
& c_{w h}\left(\tilde{b}_{(j, 2)}\right)=0 \quad \Longrightarrow \quad \alpha_{1 j} \alpha_{22}^{2}=\lambda \alpha_{2 j} \alpha_{12}^{2} .
\end{aligned}
$$

Suppose first that $\alpha_{22}=0$. Then the second equation above implies that $\alpha_{12} \alpha_{21}^{2}=$ $\lambda \neq 0$. The fourth equation gives $\alpha_{2 j}=0$, and then the third gives $\alpha_{1 j}=0$, for all $j \geq 3$. Thus $h \in M_{1,2}$ and $h$ does not fix $\left\langle b_{2}\right\rangle$. It follows that $h$ interchanges $\left\langle b_{1}\right\rangle$ and $\left\langle b_{2}\right\rangle$, and hence $\alpha_{11}=0$, and the matrix $h$ induces on $\left\langle b_{1}, b_{2}\right\rangle$ (relative to the basis $\left.\left\{b_{1}, b_{2}\right\}\right)$ is

$$
\left(\begin{array}{cc}
0 & \lambda \alpha_{21} \\
\alpha_{21} & 0
\end{array}\right)
$$

Similarly if $\alpha_{11}=0$ then $h$ fixes $\left\langle b_{1}, b_{2}\right\rangle$ setwise and induces this matrix on it. Suppose then that both $\alpha_{11}$ and $\alpha_{22}$ are non-zero. If both of $\alpha_{12}, \alpha_{21}$ are non-zero, then (4) implies that $\alpha_{22}=\lambda \alpha_{12}$ and $\alpha_{21}=\lambda \alpha_{11}$, and substituting in the first equation yields $\lambda=0$, a contradiction. Thus one of $\alpha_{12}, \alpha_{21}$ is zero. Without loss 
of generality suppose that $\alpha_{12}=0$. Evaluating the fourth, and then the third of the equations above we find that $\alpha_{1 j}=\alpha_{2 j}=0$ for all $j \geq 3$. Thus again $h \in M_{1,2}$. It follows that $M \leq M_{1,2}$, and maximality of $M$ implies that $M=M(\lambda)=M_{1,2}$ for all $\lambda$. Let $h \in M_{1,2} \backslash\left(M_{1} \cap M_{2}\right)$ so that $h$ interchanges $\left\langle b_{1}\right\rangle$ and $\left\langle b_{2}\right\rangle$. Then we have that $\alpha_{12}=\lambda \alpha_{21} \neq 0$, and this must hold for all $\lambda \in \mathrm{GF}(4)^{\#}$. This is impossible, and thus the proof is complete.

Thus to complete the analysis of the case where $H_{0}=\operatorname{Soc}(H)$ is of Lie type in characteristic 2, we must deal with such groups $H$ defined over GF(2) and acting absolutely irreducibly on a GF(2) $H$-module $V=V_{0}$, with $V_{0}$ as in Lemma 4.3. It follows from the proof of [13, Proposition 5.4.6] and [13, Remark 5.4.7 (a)], that for every case apart from $H_{0}={ }^{2} F_{4}(2)^{\prime}$, the module $V_{0}$ is one of the so-called 2-restricted highest weight modules - the 'basic building blocks' for absolutely irreducible modules for these groups, see [9]. In the exceptional case where $H_{0}={ }^{2} F_{4}(2)^{\prime}$, there are exactly two non-trivial absolutely irreducible representations of $H$ that are realisable over GF(2), and these have degrees 26 and 246, see [12, p.188]. We do not need any details of the theory of even characteristic representations; we only use the list of small-dimensional representations and their weights given in [21].

Next we examine, for classical groups, some small-dimensional modules defined over GF(2): the alternating square (in Lemma 4.5) and the adjoint (in Lemma 4.6) of the natural module. The $H$-actions on these modules are explained carefully in the proofs.

Lemma 4.5. If $H$ is a classical group defined over $\mathrm{GF}(2)$ and $V$ is the alternating square module for $H$, then $G$ is extremely primitive if and only if $d, \operatorname{Soc}(H)$ occur in one of the columns of Table 1. These examples occur in Theorems 1.2 (b) or 1.3 (b), (c).

Table 1. Table for Lemma 4.5.

\begin{tabular}{c|c|c|c|c}
\hline$d$ & 6 & 4 & 4 & 6 \\
\hline $\operatorname{Soc}(H)$ & $\operatorname{SL}(4,2)$ & $\mathrm{Sp}(4,2)^{\prime}$ & $\Omega^{-}(4,2)$ & $\operatorname{PSU}(4,2)$ \\
$\cong$ & $A_{8}$ & $A_{6}$ & $A_{5}$ & $\Omega^{-}(6,2)$ \\
\hline
\end{tabular}

Proof. Case 1: $\operatorname{Soc}(H)=\operatorname{SL}(n, 2)$ for some $n \geq 4$. Let $B=\left\{b_{1}, \ldots, b_{n}\right\}$ be a basis for the natural module $W$ of $\operatorname{Soc}(H)$. A basis of the alternating square module $W^{\wedge 2}$ is the set $B^{\wedge 2}:=\left\{b_{i} \wedge b_{j} \mid 1 \leq i<j \leq n\right\}$. If some $h \in \operatorname{Soc}(H)$ has matrix $h=\left(\alpha_{i j}\right)$ relative to $B$ then the action of $h$ on $B^{\wedge 2}$ is defined by

$$
b_{i} \wedge b_{j} \mapsto \sum_{k<l}\left(\alpha_{i, k} \alpha_{j, l}+\alpha_{i, l} \alpha_{j, k}\right) b_{k} \wedge b_{l} .
$$

If $n=4$ then the actions of $\operatorname{SL}(4,2) \cong A_{8}$ and $\operatorname{SL}(4,2) .2 \cong S_{8}$ on the alternating square module are isomorphic to their actions on the deleted permutation module and 
we get extremely primitive examples (see Lemma 4.2). Suppose now that $n>4$. In this case $\operatorname{SL}(n, 2) .2$ does not act on $W^{\wedge 2}$ and so $H=\operatorname{Soc}(H)$. We prove that the stabilizer $M_{14,23}$ of $b_{1} \wedge b_{4}+b_{2} \wedge b_{3}$ is not maximal in $H$, and hence that $G$ is not extremely primitive.

Let $h \in M_{14,23}$ with $h=\left(\alpha_{i j}\right)$ relative to $B$. We examine the entries $\alpha_{i k}$ for $5 \leq k \leq n$ and $1 \leq i \leq 4$. For some fixed $k \geq 5$, let $X_{k}:=\left\{i \leq 4 \mid \alpha_{i k} \neq 0\right\}$ and $\bar{X}_{k}:=\left\{5-i \mid i \in X_{k}\right\}$. For any $\ell \neq k$, the coefficient of $b_{k} \wedge b_{\ell}$ in $\left(b_{1} \wedge b_{4}+b_{2} \wedge b_{3}\right) h$ is 0 ; hence, by (5),

$$
0=\sum_{j=1}^{4} \alpha_{5-j, k} \alpha_{j \ell}=\sum_{j \in \bar{X}_{k}} \alpha_{5-j, k} \alpha_{j \ell}=\sum_{j \in \bar{X}_{k}} \alpha_{j \ell} .
$$

Note that, if $j \in \bar{X}_{k}$, then either (i) $j \notin X_{k}$ so that $\alpha_{j k}=0$, or (ii) $j \in X_{k}$ so that both $j, 5-j \in \bar{X}_{k}$ and $\alpha_{j k}+\alpha_{5-j, k}=1+1=0$. Hence $\sum_{j \in \bar{X}_{k}} \alpha_{j k}=0$ as well. Thus, the rows of $h$ with indices in $\bar{X}_{k}$ sum to the zero vector. If $\bar{X}_{k} \neq \emptyset$ this gives a contradiction, so $\bar{X}_{k}=\emptyset$ and hence $X_{k}=\emptyset$ also. Thus $\alpha_{i k}=0$ for all $i \leq 4$ and $k \geq 5$, and hence $M_{14,23}$ fixes the subspace $U:=\left\langle b_{1}, b_{2}, b_{3}, b_{4}\right\rangle$ in its action on $W$. The subgroup of $\operatorname{GL}(U)$ induced by $M_{14,23}$ is isomorphic to $\operatorname{Sp}(4,2)$ because stabilising $b_{1} \wedge b_{4}+b_{2} \wedge b_{3}$ in the alternating square of $\operatorname{SL}(4,2)$ is equivalent to stabilising a nondegenerate alternating form on $U$. Hence $M_{14,23}$ is a proper subgroup of the stabilizer of $U$ and so is not maximal in $H$.

Case 2: $\operatorname{Soc}(H)=\operatorname{Sp}(n, 2)^{\prime}$ with $n \geq 4, n$ even. If $n=4$ then the actions of $\mathrm{Sp}(4,2)^{\prime} \cong A_{6}$ and $\mathrm{Sp}(4,2) \cong S_{6}$ on the alternating square are isomorphic to their actions on the deleted permutation module and we get extremely primitive examples. Suppose that $n>4$. In this case $H=\operatorname{Sp}(n, 2)$. We consider $H$ as a subgroup of $\operatorname{SL}(n, 2)$ acting on $W$ and $W^{\wedge 2}$ as defined in Case 1 . We choose the basis $B$ so that $\left\{b_{2 i-1}, b_{2 i}\right\}$ is a hyperbolic pair for $1 \leq i \leq n / 2$. This means that $H$ consists of those elements $h=\left(\alpha_{i j}\right) \in \operatorname{SL}(n, 2)$ that for $1 \leq i<j \leq n$ satisfy

$$
\sum_{k=1}^{n / 2} \alpha_{i, 2 k-1} \alpha_{j, 2 k}+\alpha_{i, 2 k} \alpha_{j, 2 k-1}= \begin{cases}1 & \text { if }\left\{b_{i}, b_{j}\right\} \text { is a hyperbolic pair } \\ 0 & \text { otherwise }\end{cases}
$$

and, since the invariant bilinear form $J$ of $\operatorname{Sp}(n, 2)$ satisfies $J=\left(J^{-1}\right)^{\mathrm{tr}}=J^{-1}$, we also have $\left(\alpha_{i j}\right)^{\text {tr }} \in \operatorname{Sp}(n, 2)$ and so

$$
\sum_{k=1}^{n / 2} \alpha_{2 k-1, i} \alpha_{2 k, j}+\alpha_{2 k, i} \alpha_{2 k-1, j}= \begin{cases}1 & \text { if }\left\{b_{i}, b_{j}\right\} \text { is a hyperbolic pair } \\ 0 & \text { otherwise. }\end{cases}
$$

However, $H=\operatorname{Sp}(n, 2)$ does not act on $W^{\wedge 2}$ irreducibly. For $w=\sum_{k, \ell} \lambda_{k \ell} b_{k} \wedge$ $b_{\ell} \in W^{\wedge 2}$, let $\Lambda(w):=\sum_{k=1}^{n / 2} \lambda_{2 k-1,2 k}$. Then $\widetilde{W}:=\left\{w \in W^{\wedge 2} \mid \Lambda(w)=0\right\}$ is a 
codimension 1 subspace. (Note that $\widetilde{W}$ consists of all $\sum_{k, \ell} \lambda_{k \ell} b_{k} \wedge b_{\ell}$ such that an even number of the coefficients $\lambda_{2 i-1,2 i}$ is non-zero.) This subspace is $H$-invariant, because for any $h=\left(\alpha_{i j}\right) \in H$ and $b_{i} \wedge b_{j} \in B^{\wedge 2}$, using (5) and (7), we see that $\Lambda\left(\left(b_{i} \wedge b_{j}\right) h\right)$ is equal to

$$
\sum_{k=1}^{n / 2} \alpha_{i, 2 k-1} \alpha_{j, 2 k}+\alpha_{i, 2 k} \alpha_{j, 2 k-1}= \begin{cases}1 & \text { if }\left\{b_{i}, b_{j}\right\} \text { is a hyperbolic pair } \\ 0 & \text { otherwise }\end{cases}
$$

Also the subspace $\langle u\rangle$, where $u:=\sum_{k=1}^{n / 2} b_{2 k-1} \wedge b_{2 k}$, is $H$-invariant because for any $h=\left(\alpha_{i j}\right) \in H$ and $b_{i} \wedge b_{j} \in B^{\wedge 2}$, the coefficient of $b_{i} \wedge b_{j}$ in $u h$ is

$$
\sum_{k=1}^{n / 2} \alpha_{2 k-1, i} \alpha_{2 k, j}+\alpha_{2 k, i} \alpha_{2 k-1, j}= \begin{cases}1 & \text { if }\left\{b_{i}, b_{j}\right\} \text { is a hyperbolic pair } \\ 0 & \text { otherwise }\end{cases}
$$

by (8). If $n / 2$ is odd then $\widetilde{W}$ is irreducible, $W^{\wedge 2}=\widetilde{W} \oplus\langle u\rangle$, and $\widetilde{W}$ is the alternating square module $V$. If $n / 2$ is even, then $u \in \widetilde{W}$, the factor module $\widetilde{W} /\langle u\rangle$ is irreducible, and it is the alternating square module $V$. Let $w:=b_{1} \wedge b_{4}+b_{2} \wedge b_{3} \in \widetilde{W}$, and let $v=w$ if $n / 2$ is odd and $v=w+\langle u\rangle$ if $n / 2$ is even. So $v \in V$. We shall prove that the stabilizer $M_{14,23}$ of $v$ is not maximal in $H$ and so $G$ is not extremely primitive.

If $n / 2$ is odd then $M_{14,23}$ consists of those $h \in H$ that stabilise $w$. If $n / 2$ is even then $M_{14,23}$ is the union of those $h \in H$ that stabilise $w$ and those $h \in H$ that map $w$ to $w+u$. In either case those $h \in H$ that stabilise $w$ were shown in Case 1 to stabilise $U=\left\langle b_{1}, b_{2}, b_{3}, b_{4}\right\rangle \leq W$.

We claim that, if $n / 2$ is even, then there is no $h \in H$ which maps $w$ to $w+u$. Suppose, on the contrary, that there is an $h=\left(\alpha_{i j}\right) \in H$ such that $w h=w+u$. Let $k \geq 5$ be fixed and let $k^{\prime} \in\{k-1, k+1\}$ be such that $\left\{b_{k}, b_{k^{\prime}}\right\}$ is a hyperbolic pair. We define $X_{k}$ and $\bar{X}_{k}$ as Case 1 . Now $X_{k} \neq \emptyset$ as otherwise the coefficient of $b_{k} \wedge b_{k^{\prime}}$ would be 0 in $w h$ by (5). Also,(6) holds for all $\ell \notin\left\{k, k^{\prime}\right\}$ because the coefficient of $b_{k} \wedge b_{l}$ is 0 in $w+u$.

We consider the possibilities for $X_{k}$. If $\left|X_{k}\right|=1$, by symmetry say $X_{k}=\{1\}$, then by the definition of $X_{k}, \alpha_{3 k}=\alpha_{4 k}=0$ and, by (6), $\alpha_{4, \ell}=0$ for all $\ell \neq k^{\prime}$. This gives a contradiction to (7) applied with $i=3$ and $j=4$. Hence $\left|X_{k}\right| \geq 2$ for all $k \geq 5$. Suppose next that $\left|X_{k}\right|=4$. Then since the coefficient of $b_{k} \wedge b_{k^{\prime}}$ in $w+u$ is 1 and in $w h$ is $\sum_{i=1}^{4} \alpha_{i k^{\prime}},\left|X_{k^{\prime}}\right|$ must be odd. Since $\left|X_{k^{\prime}}\right| \geq 2$, we must have $\left|X_{k^{\prime}}\right|=3$ and by symmetry we may assume $X_{k^{\prime}}=\{1,2,3\}$. Applying (6) with $k$ and $k^{\prime}$, we obtain that for all $\ell \notin\left\{k, k^{\prime}\right\}$ the sums $\sum_{j=1}^{4} \alpha_{j \ell}=0$ and $\sum_{j=2}^{4} \alpha_{j \ell}=0$. Hence $\alpha_{1, \ell}=0$. This, combined with the fact $\alpha_{1, k}=\alpha_{2, k}=\alpha_{1, k^{\prime}}=\alpha_{2, k^{\prime}}=1$, yields a contradiction by (7) applied with $i=1$ and $j=2$. Hence $2 \leq\left|X_{k}\right| \leq 3$ for all $k \geq 5$.

Suppose next that $\left|X_{k}\right|=3$. By symmetry we may assume $X_{k}=\{1,2,3\}$. Then the coefficient of $b_{k} \wedge b_{k^{\prime}}$ in $w+u$ is 1 and in $w h$ is $\alpha_{2 k^{\prime}}+\alpha_{3 k^{\prime}}+\alpha_{4 k^{\prime}}$, and hence 
$\left|X_{k^{\prime}} \cap\{2,3,4\}\right|$ is odd. Suppose first that $\left|X_{k^{\prime}}\right|=3$. Then $\left|X_{k^{\prime}} \cap\{2,3,4\}\right| \geq 2$ and so $X_{k^{\prime}}=\{2,3,4\}$. Applying (6) with $k$ and $k^{\prime}$, we obtain that for all $\ell \notin\left\{k, k^{\prime}\right\}$ the sums $\sum_{j=2}^{4} \alpha_{j \ell}=0$ and $\sum_{j=1}^{3} \alpha_{j \ell}=0$. Adding these two equations, we obtain $\alpha_{1 \ell}+\alpha_{4 \ell}=0$, that is, $\alpha_{1 \ell}=\alpha_{4 \ell}$. Now (7) yields a contradiction for $i=1$ and $j=4$. Thus, by the previous paragraph, we must have $\left|X_{k^{\prime}}\right|=2$, and hence $\left|X_{k^{\prime}} \cap\{2,3,4\}\right|=1$ (as it is odd). If $X_{k^{\prime}}=\{1,4\}$ then applying (6) with $k^{\prime}$, we obtain $\alpha_{1 \ell}=\alpha_{4 \ell}$ for all $\ell \notin\left\{k, k^{\prime}\right\}$ and then (7) yields a contradiction for $i=1$ and $j=4$. If $X_{k^{\prime}}=\{1,2\}$ then applying (6) with $k^{\prime}$ we obtain $\alpha_{3 \ell}=\alpha_{4 \ell}$ for all $\ell \notin\left\{k, k^{\prime}\right\}$ and then (7) yields a contradiction for $i=3$ and $j=4$. Finally if $X_{k^{\prime}}=\{1,3\}$ then applying (6) with $k$ and $k^{\prime}$ we obtain that, for all $\ell \notin\left\{k, k^{\prime}\right\}$, the sum $\sum_{j=2}^{4} \alpha_{j \ell}=0$ and $\alpha_{2 \ell}=\alpha_{4 \ell}$. Hence $\alpha_{3 \ell}=0$ and (7) yields a contradiction for $i=3$ and $j=4$.

Thus the only possibility remaining is that $\left|X_{k}\right|=2$ for all $k \geq 5$. Let $\bar{X}_{k}=\{i, j\}$ and set $\{s, t\}:=\{1,2,3,4\} \backslash \bar{X}_{k}$. In this case we also fix an $m \geq 5$, with $m \notin\left\{k, k^{\prime}\right\}$ (since $n / 2>2$ and $n / 2$ is even, such an $m$ exists). Applying (6) with $k$, we obtain that $\alpha_{i m}=\alpha_{j m}$, and then also $\alpha_{s m}=\alpha_{t m}$ because $\left|X_{m}\right|=2$. Applying (6) with $k^{\prime}$, we obtain that $\alpha_{p m}=\alpha_{r m}$ for $p, r \in \bar{X}_{k^{\prime}}$. This pair $\{p, r\}$ is different from $\{i, j\}$ and $\{s, t\}$ because otherwise the coefficient of $b_{k} \wedge b_{k^{\prime}}$ would be 0 in $v$. Hence all of the first four entries in column $m$ of $H$ are equal, contradicting $\left|X_{m}\right|=2$. Thus there are no possibilities for $X_{k}$.

Summarizing, we have shown that the stabilizer $M_{14,23}$ of $v$ fixes the subspace $U=\left\langle b_{1}, b_{2}, b_{3}, b_{4}\right\rangle \leq W$. The group induced by $M_{14,23}$ on $U$ is the intersection of two symplectic groups $\operatorname{Sp}(4,2)$ (one is the stabilizer of $U$ in $H$, the other one is the stabilizer of $w \in W^{\wedge 2}$ in $\operatorname{SL}(n, 2)$ acting on $W$ ). Computation in GAP shows that the group induced by $M_{14,23}$ on $U$ is isomorphic to $S_{4} \times Z_{2}$. Hence $M_{14,23}$ is a proper subgroup of the stabilizer of $U$ in $H$ and so is not maximal in $H$.

Case 3: $\operatorname{Soc}(H)=\Omega^{ \pm}(n, 2)$ with $n$ even, $n \geq 4$. For $n=4$, since $H$ is almost simple, $\operatorname{Soc}(H)=\Omega^{-}(4,2) \cong \operatorname{SL}(2,4) \cong A_{5}$. The action of $H$ on the alternating square module is isomorphic to the deleted permutation module action of $A_{5}$ or $S_{5}$, and hence $G$ is extremely primitive. Suppose now that $n \geq 6$. In this case, $H$ is a subgroup of $\operatorname{Sp}(n, 2)$, and in its action on $W$ we can choose the basis $B$ such that the group induced by $H$ on the subspace $U$ above is $\mathrm{SO}^{+}(4,2)$. The alternating square module $V$ for $H$ is $\widetilde{W}$ if $n / 2$ is odd and $\widetilde{W} /\langle u\rangle$ if $n / 2$ is even. Again, we consider the stabilizer $M_{14,23}$ of $v$. Since $H \leq \operatorname{Sp}(n, 2)$, we know that $M_{14,23}=H_{w}$ and fixes $U \leq W$. The group induced by $M_{14,23}$ on $U$ is the intersection of an orthogonal group of plus type and a symplectic group; computation in GAP shows that it is isomorphic to $\operatorname{SL}(2,2)$. Hence $M_{14,23}$ is a proper subgroup of the stabilizer of $U$ in $H$ and so is not maximal in $H$.

Case 4: $\operatorname{Soc}(H)=\operatorname{PSU}(n, 2)$ with $n \geq 4$. If $n=4$ then the alternating square module of $\operatorname{PSU}(4,2) \cong \Omega^{-}(6,2)$ is isomorphic to the natural module of $\Omega^{-}(6,2)$, yielding two extremely primitive examples. For $n>4$, the alternating square module 
of $\operatorname{PSU}(n, 2)$ is not defined over $\mathrm{GF}(2)$. (It is defined over $\mathrm{GF}(4)$ and not $\mathrm{GF}(2)$ because its 2-restricted highest weight is $\omega_{2}$ which is not invariant under the graph automorphism of the Dynkin diagram.) Thus by Lemma 4.4, and the discussion following it, $G$ is not extremely primitive.

Lemma 4.6. Let $\operatorname{Soc}(H)=\operatorname{SL}(n, 2)$ or $\operatorname{PSU}(n, 2)$ and let $V$ be the adjoint module of $H$. Then $G$ is not extremely primitive.

Proof. Case 1: $\operatorname{Soc}(H)=\operatorname{SL}(n, 2)$ with $n \geq 3$. Suppose that $B=\left\{b_{1}, \ldots, b_{n}\right\}$ is a basis for the natural module $W$ of $\operatorname{SL}(n, 2)$ and let $W^{*}$ be the dual of $W$ with basis $B^{*}=\left\{b_{1}^{*}, \ldots, b_{n}^{*}\right\}$. For $h \in \operatorname{Soc}(H)$, if the matrix for $h$ on $W$, written relative to the basis $B$, is $h=\left(\alpha_{i j}\right)$ then the matrix of $h$ on $W^{*}$, written relative to $B^{*}$, is $\left(\alpha_{i j}^{*}\right)$, where $\left(\alpha_{i j}^{*}\right)$ is the inverse transpose of $\left(\alpha_{i j}\right)$. The connection between $\left(\alpha_{i j}\right)$ and $\left(\alpha_{i j}^{*}\right)$ is given by

$$
\sum_{k=1}^{n} \alpha_{i, k} \alpha_{j, k}^{*}= \begin{cases}1 & \text { if } i=j \\ 0 & \text { if } i \neq j\end{cases}
$$

and

$$
\sum_{k=1}^{n} \alpha_{k, i} \alpha_{k, j}^{*}= \begin{cases}1 & \text { if } i=j \\ 0 & \text { if } i \neq j .\end{cases}
$$

We consider the $n^{2}$-dimensional vector space $W \otimes W^{*}$, with basis $B \otimes B^{*}:=$ $\left\{b_{i} \otimes b_{j}^{*} \mid 1 \leq i, j \leq n\right\}$. For $h \in H$ with matrix $\left(\alpha_{i j}\right)$ relative to $B$, the action of $h$ on $W \otimes W^{*}$ is defined by

$$
b_{i} \otimes b_{j}^{*} \mapsto \sum_{k, \ell} \alpha_{i, k} \alpha_{j, \ell}^{*} b_{k} \otimes b_{\ell}^{*}
$$

If $H=\operatorname{SL}(n, 2) .2$ and $\tau \in H$ conjugates all $h=\left(\alpha_{i j}\right) \in \operatorname{Soc}(H)$ to $h^{\tau}=\left(\alpha_{i j}^{*}\right)$ then $\tau$ acts on $B \otimes B^{*}$, and hence on $W \otimes W^{*}$ by

$$
b_{i} \otimes b_{j}^{*} \mapsto b_{j} \otimes b_{i}^{*} .
$$

The group $H$ does not act on $W \otimes W^{*}$ irreducibly. For $w=\sum_{k, \ell} \lambda_{k \ell} b_{k} \otimes b_{\ell}^{*} \in$ $W \otimes W^{*}$, let $\Lambda(w):=\sum_{k=1}^{n} \lambda_{k, k}$. Then $\widetilde{W}:=\left\{w \in W \otimes W^{*} \mid \Lambda(w)=0\right\}$ is a codimension 1 subspace. This subspace is $H$-invariant, because $\Lambda(w \tau)=\Lambda(w)$ for any $w$, and for any $h=\left(\alpha_{i j}\right) \in \operatorname{Soc}(H), \Lambda(w h)=\sum_{i, j} \lambda_{i j} \Lambda\left(\left(b_{i} \otimes b_{j}\right) h\right)$, and by (11) and (9),

$$
\Lambda\left(\left(b_{i} \otimes b_{j}^{*}\right) h\right)=\sum_{k=1}^{n} \alpha_{i, k} \alpha_{j, k}^{*}= \begin{cases}1 & \text { if } i=j \\ 0 & \text { if } i \neq j\end{cases}
$$


Let $u:=\sum_{k=1}^{n} b_{k} \otimes b_{k}^{*}$. Then $\langle u\rangle$ is $H$-invariant because $u \tau=u$ and, for any $h=\left(\alpha_{i j}\right) \in \operatorname{Soc}(H)$ and $b_{i} \otimes b_{j}^{*} \in B \otimes B^{*}$, the coefficient of $b_{i} \otimes b_{j}^{*}$ in $u h$ is

$$
\sum_{k=1}^{n} \alpha_{k, i} \alpha_{k, j}^{*}= \begin{cases}1 & \text { if } i=j \\ 0 & \text { if } i \neq j\end{cases}
$$

by (10). If $n$ is odd then $\widetilde{W}$ is irreducible, $W \otimes W^{*}=\widetilde{W} \oplus\langle u\rangle$, and $\widetilde{W}$ is the adjoint module $V$. If $n$ is even then $u \in \widetilde{W}$ and the factor module $\widetilde{W} /\langle u\rangle$ is irreducible, called the adjoint module $V$. Let $w:=b_{1} \otimes b_{2}^{*}+b_{2} \otimes b_{1}^{*} \in \widetilde{W}$ and let $v=w$ if $n$ is odd, and $v=w+\langle u\rangle$ if $n$ is even, so in both cases $v \in V$. We shall prove that in either case the stabilizer $M_{12,21}$ of $v$ is not maximal in $H$ and so $G$ is not extremely primitive.

If $n$ is odd then $M_{12,21}$ consists of those $h \in H$ that stabilise $w$. If $n$ is even then $M_{12,21}$ is the union of those $h \in H$ that stabilise $w$ and those that map $w$ to $w+u$. Since by (12), $w \tau=w$, we only need to consider elements of $\operatorname{Soc}(H)_{v}$. Suppose first that $h=\left(\alpha_{i j}\right) \in \operatorname{Soc}(H)$ stabilises $w$. For $k \geq 3$, let $X_{k}:=\left\{i \leq 2 \mid \alpha_{i k} \neq 0\right\}$ and $\bar{X}_{k}:=\left\{3-i \mid i \in X_{k}\right\}$. Similarly, let $X_{k}^{*}:=\left\{i \leq 2 \mid \alpha_{i k}^{*} \neq 0\right\}$ and $\bar{X}_{k}^{*}:=\left\{3-i \mid i \in X_{k}^{*}\right\}$. For any $\ell \leq n$, the coefficient of $b_{k} \otimes b_{\ell}^{*}$ in $w h$ is 0 . Hence, by (11), $\sum_{j \in \bar{X}_{k}} \alpha_{3-j, k} \alpha_{j \ell}^{*}=0$. Thus if $X_{k} \neq \emptyset$ then a nontrivial linear combination of the rows of $\left(\alpha_{i j}^{*}\right)$ is the zero vector, which is a contradiction. Similarly, for any $\ell \leq n$ the coefficient of $b_{\ell} \otimes b_{k}^{*}$ in $w h$ is 0 . Hence $\sum_{j \in \bar{X}_{k}^{*}} \alpha_{3-j, \ell} \alpha_{j k}^{*}=0$, and if $X_{k}^{*} \neq \varnothing$ then a nontrivial linear combination of the rows of $h$ is the zero vector, which is a contradiction. Hence $\alpha_{i k}=\alpha_{i k}^{*}=0$, for $1 \leq i \leq 2,3 \leq k \leq m$. Since $\left(\alpha_{i j}^{*}\right)=\left(h^{-1}\right)^{\mathrm{tr}}$, this implies that also $\alpha_{k i}=0$, for $1 \leq i \leq 2,3 \leq k \leq m$. Thus $h$ fixes the subspaces $U:=\left\langle b_{1}, b_{2}\right\rangle$ and $U^{\prime}:=\left\langle b_{3}, \ldots, b_{n}\right\rangle$ in its action on $W$, and hence $\operatorname{Soc}(H)_{w} \leq \operatorname{Soc}(H)_{U, U^{\prime}}$.

We claim that, when $n$ is even, no $h \in \operatorname{Soc}(H)$ maps $w$ to $w+u$. Suppose on the contrary that $h=\left(\alpha_{i j}\right)$ is such an element. For $k \geq 3$, we define $X_{k}$ and $X_{k}^{*}$ as above. Now $X_{k} \neq \varnothing$ and $X_{k}^{*} \neq \varnothing$ because otherwise the coefficient of $b_{k} \otimes b_{k}^{*}$ in $w h$ would be 0 . Suppose that $\left|X_{k}\right|=1$, say $X_{k}=\{a\}$. Then by (11), for any $\ell \neq k$, the coefficient of $b_{k} \otimes b_{\ell}^{*}$ in $w h$ is $\alpha_{3-a, \ell}$ and this must be 0 . Choose an $m \geq 3$, $m \neq k$ (such an $m$ exists because $n>2$ and $n$ is even). We have $\alpha_{3-a, m}^{*}=0$ and $X_{m}^{*} \neq \emptyset$, so $\alpha_{a, m}^{*} \neq 0$. Then (11) implies that $\alpha_{3-a, \ell}=0$ for all $\ell \neq m$. However, this contradicts (9) for $i=j=3-a$. Thus $X_{k}=\{1,2\}$ for all $k \geq 3$. Reversing the role of $\left(\alpha_{i j}\right)$ and $\left(\alpha_{i j}^{*}\right)$, the same argument proves that $X_{k}^{*}=\{1,2\}$ for all $k \geq 3$. Hence the coefficient of $b_{k} \otimes b_{k}^{*}$ in $w h$ is $\alpha_{1 k} \alpha_{2 k}^{*}+\alpha_{2 k} \alpha_{1 k}^{*}=1+1=0$, which is a contradiction. This proves the claim.

For later use, we emphasize that the argument in the previous two paragraphs did not use the fact that the $\alpha_{i j}$ are elements of GF(2) until the very last step (to reach a contradiction in the case $\left|X_{k}\right|=\left|X_{k}^{*}\right|=2$ for all $k \geq 3$ ). Instead, it utilized only the fact that the $\alpha_{i j}$ satisfy (9)-(11). So far we have proved that $M_{12,21} \cap \operatorname{Soc}(H)$ fixes 
$U \leq W$ and $U^{\prime} \leq W$. As noted above $w \tau=w$. Moreover, since $h^{\tau}=\left(h^{-1}\right)^{\text {tr }}$ for $h \in \operatorname{Soc}(H), \tau$ normalizes $\operatorname{Soc}(H)_{U, U^{\prime}}$. An easy computation in $\operatorname{SL}(2,2)$ shows that the group induced by $M_{12,21} \cap \operatorname{Soc}(H)$ on $U$ is isomorphic to $Z_{2}$. Hence $M_{12,21} \cap$ $\operatorname{Soc}(H)$ is a proper subgroup of $\operatorname{Soc}(H)_{U, U^{\prime}}$. If $H=\operatorname{Soc}(H)$ then $M_{12,21}<$ $H_{U, U^{\prime}}<H$, while if $H=\operatorname{Soc}(H) .\langle\tau\rangle$ then $M_{12,21}<\operatorname{Soc}(H)_{U, U^{\prime}} \cdot\langle\tau\rangle<H$, and so in either case $M_{12,21}$ is not maximal in $H$.

Case 2: $\operatorname{Soc}(H)=\operatorname{PSU}(n, 2)$ with $n \geq 4$. Generators for the adjoint module of PSU $(4,2) .2$ are available in at lasrep and from these we also constructed generators for PSU $(4,2)$. We computed the orbits of these groups and checked that they do not act primitively on all orbits.

Suppose that $n \geq 5$ and let $W$ be the natural module of $\operatorname{SL}(n, 4)$ with basis $B=\left\{b_{1}, \ldots, b_{n}\right\}$. For $h=\left(\alpha_{i j}\right) \in \operatorname{SL}(n, 4)$, we define $\bar{h}:=\left(\alpha_{i j}^{2}\right)$. The group $\mathrm{SU}(n, 2)$ in its action on $W$ is defined as the subgroup of all matrices $h \in \operatorname{SL}(n, 4)$ satisfying

$$
h^{-1}=\bar{h}^{\mathrm{tr}} .
$$

We define $W^{*}, B^{*}, W \otimes W^{*}, B \otimes B^{*}$, and $\left(\alpha_{i j}^{*}\right)$ as in Case 1 . The elements $\alpha_{i j}, \alpha_{i j}^{*} \in$ $\mathrm{GF}(4)$ satisfy (9) and (10). In addition, because of (13), if $h=\left(\alpha_{i j}\right) \in \mathrm{SU}(n, 2)$ then for all $i, j$

$$
\alpha_{i j}^{*}=\alpha_{i j}^{2} .
$$

The action of $\operatorname{Soc}(H)$ on $W \otimes W^{*}$ is defined by (11). If the group $H=\operatorname{PSU}(n, 2) .2$ and $\sigma \in H$ conjugates all $h \in \operatorname{Soc}(H)$ to $h^{\sigma}=\bar{h}$ then by (14), $h^{\sigma}$ is the inverse transpose of $h$. Hence the action of $\sigma$ on $W \otimes W^{*}$ is defined, see (12), by $\sum_{i, j} \lambda_{i j} b_{i} \otimes$ $b_{j}^{*} \mapsto \sum_{i, j} \lambda_{i j}^{2} b_{j} \otimes b_{i}^{*}$. We define $\widetilde{W}$ and $u$ as above and the same argument as in Case 1 yields that $\widetilde{W}$ and $\langle u\rangle$ are $H$-invariant. Moreover, we define a GF(2)-subspace $\widetilde{W}_{0}$ by

$$
\widetilde{W}_{0}:=\left\{w=\sum_{i, j} \lambda_{i j} b_{i} \otimes b_{j}^{*} \in \widetilde{W} \mid \lambda_{j i}=\lambda_{i j}^{2} \text { for all } i, j\right\} .
$$

Let $\omega$ be a generator of GF(4)* Then $\widetilde{W}_{0}$ has a GF(2)-basis $\mathbb{B}$ of size $n^{2}-1$ consisting of the $\left(\begin{array}{c}n \\ 2\end{array}\right)$ vectors $b_{i} \otimes b_{j}^{*}+b_{j} \otimes b_{i}^{*}$ for $i<j$, the $\left(\begin{array}{c}n \\ 2\end{array}\right)$ vectors $\omega b_{i} \otimes b_{j}^{*}+\omega^{2} b_{j} \otimes b_{i}^{*}$ for $i<j$, and the $n-1$ vectors $b_{1} \otimes b_{1}^{*}+b_{i} \otimes b_{i}^{*}$ for $i \geq 2$. We claim that $\widetilde{W}_{0}$ is $H$-invariant. If $h=\left(\alpha_{i j}\right) \in \operatorname{Soc}(H)$ then by (11) for any $i, j, k, \ell$ the coefficients of $b_{k} \otimes b_{\ell}^{*}$ and $b_{\ell} \otimes b_{k}^{*}$ in $\left(b_{i} \otimes b_{j}^{*}+b_{j} \otimes b_{i}^{*}\right) h$ are $\alpha_{i k} \alpha_{j \ell}^{*}+\alpha_{j k} \alpha_{i \ell}^{*}$ and $\alpha_{i \ell} \alpha_{j k}^{*}+$ $\alpha_{j \ell} \alpha_{i k}^{*}$, respectively and by (14) these coefficients are the squares of each other. Thus $\left(b_{i} \otimes b_{j}^{*}+b_{j} \otimes b_{i}^{*}\right) h \in \widetilde{W}_{0}$. Similarly, the coefficients of $b_{k} \otimes b_{\ell}^{*}$ and $b_{\ell} \otimes b_{k}^{*}$ in $\left(\omega b_{i} \otimes b_{j}^{*}+\omega^{2} b_{j} \otimes b_{i}^{*}\right) h$ are $\omega \alpha_{i k} \alpha_{j \ell}^{*}+\omega^{2} \alpha_{j k} \alpha_{i \ell}^{*}$ and $\omega \alpha_{i \ell} \alpha_{j k}^{*}+\omega^{2} \alpha_{j \ell} \alpha_{i k}^{*}$, respectively and by (14) these coefficients are the squares of each other. Finally, the coefficients of $b_{k} \otimes b_{\ell}^{*}$ and $b_{\ell} \otimes b_{k}^{*}$ in $\left(b_{1} \otimes b_{1}^{*}+b_{i} \otimes b_{i}^{*}\right) h$ are $\alpha_{1 k} \alpha_{1 \ell}^{*}+\alpha_{i k} \alpha_{i \ell}^{*}$ and $\alpha_{1 \ell} \alpha_{1 k}^{*}+\alpha_{i \ell} \alpha_{i k}^{*}$, respectively and by (14) these coefficients are the squares of each other. Thus $h$ leaves $\widetilde{W}_{0}$ invariant. Moreover, $\sigma$ fixes the basis vectors 
$b_{i} \otimes b_{j}^{*}+b_{j} \otimes b_{i}^{*}$ and $b_{1} \otimes b_{1}^{*}+b_{i} \otimes b_{i}^{*}$, and maps $\omega b_{i} \otimes b_{j}^{*}+\omega^{2} b_{j} \otimes b_{i}^{*}$ to $\omega^{2} b_{i} \otimes b_{j}^{*}+\omega b_{j} \otimes b_{i}^{*}=\left(b_{i} \otimes b_{j}^{*}+b_{j} \otimes b_{i}^{*}\right)+\left(\omega b_{i} \otimes b_{j}^{*}+\omega^{2} b_{j} \otimes b_{i}^{*}\right) \in \widetilde{W}_{0}$. Thus $\sigma$ fixes $\widetilde{W}_{0}$, and hence $\widetilde{W}_{0}$ is $H$-invariant, as claimed.

The adjoint module $V$ of $H$ is defined as the GF(2)-module $\widetilde{W}_{0}$ if $n$ is odd, and as $\widetilde{W}_{0} /\langle u\rangle_{\mathrm{GF}(2)}$ if $n$ is even. Define $w, u$, and $v \in V$ as in Case 1 . We claim that the stabilizer $M_{12,21}$ of $v$ is not maximal in $H$ and so $G$ is not extremely primitive. Now $w \sigma=w$ (by the $\sigma$-action described above), and hence $\sigma \in H_{v}$, so we need only consider elements of $\operatorname{Soc}(H)_{v}$.

If $h \in M_{12,21} \cap \operatorname{Soc}(H)$ then repeating verbatim the argument in Case 1, we obtain that $h$ fixes $U=\left\langle b_{1}, b_{2}\right\rangle \leq W$ and $U^{\prime}=\left\langle b_{3}, \ldots, b_{n}\right\rangle \leq W$, provided we have a contradiction in the last line of proof (the case $w h=w+u$ and $\left|X_{k}\right|=\left|X_{k}^{*}\right|=2$ for all $k \geq 3$ ). In this case, fix three different indices $k, \ell, m \geq 3$ (such indices exist because $n \geq 5$ ). Note that, by the assumptions in this last case, $\alpha_{i j} \neq 0$ for any $i \leq 2$ and $j \in\{k, \ell, m\}$, and hence (using (14)), $\alpha_{i j}^{-1}=\alpha_{i j}^{2}=\alpha_{i j}^{*}$. The coefficients of $b_{k} \otimes b_{\ell}^{*}, b_{k} \otimes b_{m}^{*}, b_{\ell} \otimes b_{m}^{*}$ in $w h$ are $\alpha_{1 k} \alpha_{2 \ell}^{*}+\alpha_{2 k} \alpha_{1 \ell}^{*}=0, \alpha_{1 k} \alpha_{2 m}^{*}+\alpha_{2 k} \alpha_{1 m}^{*}=0$, and $\alpha_{1 \ell} \alpha_{2 m}^{*}+\alpha_{2 \ell} \alpha_{1 m}^{*}=0$, respectively. From the first two equations we obtain $\alpha_{1 \ell}^{2} \alpha_{2 \ell}=\alpha_{1 k} \alpha_{2 k}^{2}=\alpha_{1 m}^{2} \alpha_{2 m} \neq 0$, and from the third, $\alpha_{1 \ell} \alpha_{2 \ell}^{2}=\alpha_{1 m}^{2} \alpha_{2 m} \neq 0$. These imply that $\alpha_{1 j}=\alpha_{2 j}$ for each $j \in\{k, \ell, m\}$. Hence the coefficient of $b_{\ell} \otimes b_{\ell}^{*}$ in $w h$ is $\alpha_{1 \ell} \alpha_{2 \ell}^{*}+\alpha_{2 \ell} \alpha_{1 \ell}^{*}=0$, which is a contradiction. Thus $M_{12,21} \cap \operatorname{Soc}(H) \leq$ $\operatorname{Soc}(H)_{U, U^{\prime}}$.

The stabilizer of $U$ and $U^{\prime}$ in $\operatorname{Soc}(H)$ acts as $\operatorname{SL}(2,2)$ on $U$, with generators

$$
\left(\begin{array}{cc}
\omega & 0 \\
0 & \omega^{2}
\end{array}\right), \quad\left(\begin{array}{ll}
0 & 1 \\
1 & 0
\end{array}\right)
$$

An easy calculation shows that $M_{12,21} \cap \operatorname{Soc}(H)$ acts as $Z_{2}$ on $U$ (generated by the second matrix above). Hence $M_{12,21} \cap \operatorname{Soc}(H)$ is a proper subgroup of $\operatorname{Soc}(H)_{U, U^{\prime}}$. As noted above, $w \sigma=w$. Also, since $h^{\sigma}=\bar{h}=\left(h^{-1}\right)^{\text {tr }}$ (by (13)) for all $h \in \operatorname{Soc}(H)$, $\sigma$ normalizes $\operatorname{Soc}(H)_{U, U^{\prime}}$. If $H=\operatorname{Soc}(H)$ then $M_{12,21}<H_{U, U^{\prime}}<H$, while if $H=\operatorname{Soc}(H) \cdot\langle\sigma\rangle$ then $M_{12,21}<\operatorname{Soc}(H)_{U, U^{\prime}} \cdot\langle\sigma\rangle<H$, and so in either case $M_{12,21}$ is not maximal in $H$.

We are now ready to prove the last assertion of Theorem 1.3

Theorem 4.7. There are only finitely many finite extremely primitive groups of affine type that are not listed in Theorems 1.2 or 1.3 .

Proof. It is clear that for a fixed almost simple group $H$ there are only finitely many $d$ such that $G=Z_{2}^{d}$. $H$ is primitive, and that for a fixed $d$ there are only finitely many almost simple groups $H$ such that $G=Z_{2}^{d}$. $H$ is primitive. As a first consequence of this observation, we obtain that there are only finitely many extremely primitive groups $G=Z_{2}^{d}$. $H$ with $\operatorname{Soc}(H)$ sporadic. 
Next, for $\operatorname{Soc}(H)=A_{n}$, the groups $Z_{2}^{d}$. $H$ with $H$ acting on the deleted permutation module for the natural representation are listed in Theorems 1.2 or 1.3. By [10], if $n \geq 15$ then each faithful irreducible representation of $H$, apart from this deleted permutation module, has dimension at least $n(n-5) / 2$. Now for $n$ large enough, $n !<2^{n(n-5) / 4}$. Also, by [20], for $n$ large enough $A_{n}$ and $S_{n}$ have less than $n$ ! maximal subgroups. Hence, for large enough $n$, a primitive group $G=Z_{2}^{d} \cdot H$ with $\operatorname{Soc}(H)=A_{n}$ and $d>n$ cannot be extremely primitive by Lemma 4.1.

Suppose next that $H$ is of Lie type and the characteristic of $H$ is odd. Examining the list of bounds for the minimal dimensions of cross-characteristic representations of Lie-type groups (originally in [15] and [27], with subsequent improvements listed in [29]), we see that if $H$ acts irreducibly on $V=\mathrm{GF}(2)^{d}$ then $|H|<(2 d)^{2(2+\log d)}$. Hence, for $|H|$ large enough, for any primitive $G=Z_{2}^{d}$. $H$ we have $|H|^{8 / 5}<2^{d / 2}$. Also, by [20], for $|H|$ large enough, $H$ has less than $|H|^{8 / 5}$ maximal subgroups. Hence $G=Z_{2}^{d}$. $H$ cannot be extremely primitive by Lemma 4.1 .

Suppose that $H$ is of Lie type of even characteristic, and also (using [20]) suppose that $|H|$ is large enough such that the number of maximal subgroups of $H$ is less than $|H|^{8 / 5}$. By Lemma 4.4 and the discussion following, if $G$ is extremely primitive then $H$ is defined over $\mathrm{GF}(2)$ and, apart from one group, $V$ is a 2-restricted highest weight module for $\operatorname{Soc}(H)$. Classical groups $H$ with rank at most 55 and exceptional groups give finitely many examples. If $H$ is classical of rank $\ell \geq 55$ and $d \geq l^{3} / 8$ then $2^{d / 2}>|H|^{8 / 5}$ and $G$ is not extremely primitive by Lemma 4.1. If $\ell \geq 55$ and $d<\ell^{3} / 8$ then by [21, Theorem 5.1] the only possibilities for $V$ are the natural, alternating square, and adjoint modules. The natural representation of $H$ leads to the examples in Theorem 1.2 (b) (i) and Theorem 1.3 (c). Moreover, Lemmas 4.5 and 4.6 imply that there are only finitely many extremely primitive groups obtained from the alternating square and adjoint representations.

This completes the proof of Theorem 1.3.

\subsection{Commentary on completing the classification of affine extremely primitive} groups. The unknown exceptions in Theorem 4.7 are due to the fact that we do not have a good bound on the number of maximal subgroups that is valid in all almost simple groups. A famous conjecture of G. E. Wall states that the number of maximal subgroups is at most $|H|$ in all groups $H$. The result of [20] quoted in the proof of Theorem 4.7 says that Wall's conjecture holds for $A_{n}$ and $S_{n}$ for large enough $n$. We also know that if $\operatorname{Soc}(H)$ is sporadic then $H$ satisfies Wall's conjecture. Now consider the remaining case where $\operatorname{Soc}(H)$ is a Lie type simple group of rank $r$ over a field of order $q$. It was proved recently by M. W. Liebeck, B. M. S. Martin and A. Shalev (see Theorem 1.3 of [18], together with the discussion following it) that the number of conjugacy classes of maximal subgroups of $H$ is at most $c r^{r} \log \log q$ for some constant $c$. This number is at most $|H|^{o(1)}$, and each conjugacy class has size less than $|H|$. Hence the number of maximal subgroups of $H$ is at most $|H|^{1+o(1)}$. 
In anticipation of a full proof of Wall's conjecture for almost simple groups, we prove a stronger version of Theorem 4.7. Let $\&$ be the family of almost simple groups satisfying Wall's conjecture.

Theorem 4.8. If $H \in \delta$ and $G=Z_{2}^{d}$. $H$ is extremely primitive then either $G$ is listed in Theorems 1.2 or 1.3 , or $(d, \operatorname{Soc}(H))$ is as in one of the lines of Table 2.

Proof. We do a refined version of the proof of Theorem 4.7.

Table 2. Table for Theorem 4.8.

\begin{tabular}{cll}
\hline$d$ & $\operatorname{Soc}(H)$ & Conditions \\
\hline 40 & $\operatorname{PSp}(4,9), \operatorname{SL}(5,2)$ & $\begin{array}{l}\text { two non-permutationally } \\
\text { isomorphic groups for SL(5, 2) }\end{array}$ \\
48 & $\operatorname{Sp}(8,2), \Omega^{ \pm}(8,2)$ & \\
70 & $\mathrm{SL}(8,2), \operatorname{PSU}(8,2)$ & \\
100 & $\mathrm{Sp}(10,2)$ & \\
126 & $\mathrm{SL}(9,2)$ & $7 \leq k \leq 14$ \\
$\left(\begin{array}{l}k \\
3\end{array}\right)$ & $\mathrm{SL}(k, 2)$ & \\
$2^{k}$ & $\mathrm{Sp}(2 k, 2), \Omega^{+}(2 k+2,2)$ & $5 \leq k \leq 8$ \\
\hline 27,78 & $\mathrm{E}_{6}(2)$ & \\
78 & ${ }^{2} \mathrm{E}_{6}(2)$ & \\
56,132 & $\mathrm{E}_{7}(2)$ & \\
248 & $\mathrm{E}_{8}(2)$ & \\
\hline
\end{tabular}

Case 1: $\operatorname{Soc}(H)$ is sporadic. In this case, the maximal subgroups of $H$ are known [32], and we can use the exact number of maximal subgroups in the estimate of Lemma 4.1 to get an upper bound $d_{1}(H)$ on the dimension of the representations we have to consider. Also, the minimal dimension $d_{2}(H)$ of characteristic 2 representations is known [11]. In the twelve cases $\operatorname{Soc}(H)=\mathrm{Suz}_{2} \mathrm{Fi}_{22}, \mathrm{Fi}_{23}, \mathrm{Fi}_{24}^{\prime}, \mathrm{He}, \mathrm{HN}, \mathrm{Th}, \mathrm{O}^{\prime} \mathrm{N}, \mathrm{Ly}, J_{3}, B, M$ we have $d_{1}(H)<d_{2}(H)$ so there are no extremely primitive examples. In the remaining cases, matrices for all representations of dimension at most $d_{1}(H)$ are available in [32] and at lasrep [31]. By Lemma 3.1, $d$-dimensional representations of the form $H \leq \mathrm{GL}(d / 2,4) .2$ give no extremely primitive examples. In representations of dimension $d \leq 22$, we simply computed all orbits of $H$ on $V=\mathrm{GF}(2)^{d}$ and the permutation action of $\mathrm{H}$ on these orbits. We already described the handling of $\mathrm{Co}_{1}$ in 24 dimensions in the proof of Lemma 4.2. These computations led to the examples in Lemma 4.2. The remaining cases are $(d, H)=\left(112, J_{4}\right)$ and $(28, \mathrm{Ru})$. In these two groups, generators for representatives of all conjugacy classes of maximal subgroups are available in [32] and at lasrep, so we could compute the exact value of 
$|\operatorname{fix}(M)|$ for all maximal subgroups. This count, with an application of Lemma 4.1, proved that these remaining groups are not extremely primitive.

Case 2: $\operatorname{Soc}(H)$ is alternating. Suppose that $\operatorname{Soc}(H)=A_{n}$ and let $d_{1}(H):=$ $2\lceil\log n !\rceil$. Lemma 4.1 and our assumption $H \in \delta$ implies that if $d>d_{1}(H)$ then $G=Z_{2}^{d} . H$ is not extremely primitive. The smallest nontrivial GF(2)-representation of $H$ is the deleted permutation module, leading to extremely primitive groups in Theorems 1.2 (b) and 1.3(b). By [10], if $n \geq 15$ then the second smallest representation has dimension at least $n(n-5) / 2$. If $n \geq 17$ then $n(n-5) / 2>d_{1}(H)$ and so there are no further extremely primitive examples. For $n \leq 16$ the exact number of maximal subgroups is available, and Lemma 4.1 gives an upper bound on the dimension of representations we have to consider. We constructed the representations of dimension at most 20 and checked the orbit lengths of $H$ on $V=\mathrm{GF}(2)^{d}$, leading (only) to the examples listed in Theorem 1.3. The only cases left were $(d, H)=\left(32, S_{11}\right)$ and $\left(32, S_{12}\right)$. In these cases, $H \leq \operatorname{GL}(16,4) .2$ and so Lemma 3.1 implies that $G=Z_{2}^{d} \cdot H$ is not extremely primitive.

Case 3: $\operatorname{Soc}(H)$ is Lie type of odd characteristic. We proceed as in the proof of Theorem 4.7, just doing the estimates more precisely. In cross-characteristic representations $|H|<(2 d)^{2(2+\log d)}$ so for $d>2^{10}$ we have $|H|<2^{d / 2}$ and, by Lemma 4.1 and the assumption $H \in \mathcal{8}, G$ is not extremely primitive. There are only finitely many $H$ that have cross-characteristic representations of dimension at most $2^{10}$. Most of these groups are eliminated by computing the exact value of $|H|$ and comparing it with $2^{d / 2}$ for the minimal dimension $d$ of a cross-characteristic representation. The remaining possibilities for $\operatorname{Soc}(H)$ are $\operatorname{PSL}(2, q)$ for $11 \leq q \leq 73, \operatorname{PSL}(3,3), \operatorname{PSL}(3,5)$, $\operatorname{PSL}(4,3), \operatorname{PSp}(4, q)$ for $5 \leq q \leq 11, \operatorname{PSp}(6,3), \operatorname{PSp}(6,5), \operatorname{PSp}(8,3), \operatorname{PSp}(10,3)$, $\operatorname{PSU}(3,5), \operatorname{PSU}(3,7), \operatorname{PSU}(4,3), \operatorname{PSU}(5,3)$, and $G_{2}(3)$ (because of the isomorphisms $\operatorname{PSL}(2,5) \cong \operatorname{PSL}(2,4), \operatorname{PSL}(2,7) \cong \operatorname{PSL}(3,2), \operatorname{PSp}(4,3) \cong \operatorname{PSU}(4,2)$, and $\operatorname{PSU}(3,3) \cong G_{2}(2)^{\prime}$, we consider these groups to be defined in even characteristic and we consider $\operatorname{PSL}(2,9) \cong A_{6}$ as an alternating group).

Suppose first that $\operatorname{Soc}(H)=\operatorname{PSL}(2, q)$, for some $q$ with $11 \leq q \leq 73$. The exact number of maximal subgroups of $H$ is known, and using this number in the estimate of Lemma 4.1 we get an upper bound $d_{1}(H)$ for the dimension of representations we have to consider. In all cases, $d_{1}(H)<2(q-1)$; if $q \geq 23$ then $d_{1}(H)<q-1$; and if $q \geq 61$ then $d_{1}(H)<(q-1) / 2$. If $11 \leq q \leq 19$ then all representations of dimension less than $2(q-1)$ actually have dimension at most 20 . We have constructed all such representations (as factors of the tensor square of the permutation module of dimension $q+1$ ), computed all orbit lengths of $H$ and verified that $H$ acts primitively on each of its orbits only in the case $d=8, H=\operatorname{PSL}(2,17)$. This appears in Theorem 1.3 (c). For $q \geq 23$, the only even characteristic representation of $H$ of dimension less than $q-1$ is of dimension $(q-1) / 2$, and hence $d=(q-1) / 2$ and $q \leq 59$. If $q \equiv 3,5 \bmod 8$ then the $(q-1) / 2$-dimensional representation is over $\mathrm{GF}(4)$ which is not extremely primitive by Lemma 3.1. For $q \equiv 1,7 \bmod 8$ and 
$23 \leq q \leq 41$, we constructed the $(q-1) / 2$-dimensional representation of $\operatorname{Soc}(H)$, and computed the orbit lengths. In each case there are vectors $v \in V$ whose stabilizer is not maximal in $\operatorname{Soc}(H)$. This implies that the stabilizer of $v$ is not maximal in $H$ because for any maximal $M<H$ we have $M \cap \operatorname{Soc}(H)$ maximal in $H$. Finally, in the cases $q=47,49$, in the $(q-1) / 2$-dimensional representations we found vectors $v$ whose stabilizer is trivial in $\operatorname{Soc}(H)$ by a random search.

In the remaining cases, if $\operatorname{Soc}(H)$ is special linear, unitary, or $G_{2}(3)$ then we use the exact number of maximal subgroups of $H$ in Lemma 4.1 to get an upper bound $d_{1}(H)$ for the dimension of representations we have to consider. In the cases where $\operatorname{Soc}(H)=\operatorname{PSL}(3,5), \operatorname{PSU}(3,7)$, and $\operatorname{PSU}(5,3), d_{1}(H)$ is less than the minimal dimension of a characteristic two representation and so no extremely primitive examples arise. If $\operatorname{Soc}(H)=\operatorname{PSL}(3,3)$ or $G_{2}(3)$, the only representations to consider have dimensions 12 and 14, respectively. Generators for all possible $H$ are available in atlasrep and we computed all orbits of $H$ and found ones on which $H$ does not act primitively. If $\operatorname{Soc}(H)=\operatorname{PSL}(4,3)$ then only $d=26$ is possible. We constructed the representation of $\operatorname{Soc}(H)$ by restricting the integer representation in at lasrep to $\operatorname{GF}(2)$ and by random search found $v \in V$ whose stabilizer has order 16 . Since $\mid$ Out(PSL $(4,3)) \mid=4$, this means that the stabilizer $H_{v}$ of $v$ is a proper subgroup of a Sylow 2-subgroup of $H$ and so it is not maximal. If $\operatorname{Soc}(H)=\operatorname{PSU}(3,5), \operatorname{PSU}(4,3)$ then only $d=20$ is possible. We constructed the representation of $\operatorname{Soc}(H)$ (from the GF(2)-representation of PSU(3,5).2 and as a composition factor of the restriction of the 21-dimensional integer representation of $\operatorname{PSU}(4,3)$, respectively, both of these available in atlasrep). In the case $\operatorname{Soc}(H)=\operatorname{PSL}(3,5)$, there are vectors with stabilizers of order 2 and so their stabilizers are not maximal in $H$. In the case $\operatorname{Soc}(H)=\operatorname{PSU}(4,3)$, there are vectors with stabilizer of size 16. Since $|\operatorname{Out}(\mathrm{PSU}(4,3))|=8$, again $H_{v}$ is a proper subgroup of a Sylow 2-subgroup of $H$ and so is not maximal.

Finally, in the cases where $\operatorname{Soc}(H)=\operatorname{PSp}(2 n, q)$, [8] (see also [29, Section 4.3]) implies that the only irreducible GF(2)-representations satisfying $|H|<2^{d / 2}$ are of dimension $\left(q^{n}-1\right) / 2$ or $q^{n}-1$, the latter occurring when the $\left(q^{n}-1\right) / 2$-dimensional representations (the so-called Weyl modules) are over GF(4). However, it follows from Lemma 3.1 that the $\left(q^{n}-1\right)$-dimensional representations do not lead to extremely primitive examples. Out of the eight cases for $\operatorname{Soc}(H)=\operatorname{PSp}(2 n, q)$ under consideration, the only two where the Weyl modules are defined over GF(2) are $(d, \operatorname{Soc}(H))=(24, \operatorname{PSp}(4,7))$ and $(40, \operatorname{PSp}(4,9))$. We constructed the 24-dimensional modules for $\operatorname{PSp}(4,7)$ and in the fixed point space of an element of order 5 found a vector whose stabilizer has order 60, which cannot be maximal. The last case $(d, \operatorname{Soc}(H))=(40, \operatorname{PSp}(4,9))$ is listed in the statement of Theorem 4.8 as a candidate leading to an extremely primitive permutation group.

Case 4: $\operatorname{Soc}(H)$ is Lie type of even characteristic. As discussed after Lemma 4.4, it is enough to consider the case that $H$ is defined over $\operatorname{GF}(2)$, and either $V$ is a 
2-restricted highest weight module of $\operatorname{Soc}(H)$, or $\operatorname{Soc}(H)={ }^{2} F_{4}(2)^{\prime}$ and $\operatorname{dim} V$ is 26 or 246. As in the previous cases, we use Lemma 4.1 and our assumption that $H \in \mathcal{S}$ to obtain the upper bound $d_{1}(H):=2\lceil\log |H|\rceil$ for the dimension of representations that may lead to extremely primitive examples. If $H$ is classical of rank $n$ then $d_{1}(H)$ is a quadratic function of $n$. In particular, if $n>16$ then $d_{1}(H)<n^{3} / 8$ and if $n>11$ then $d_{1}(H)<n^{3}$. However, [21, Theorem 5.1] states that if $n>11$ then the only absolutely irreducible representations of dimension less than $n^{3} / 8$ in the special linear and unitary cases are the trivial, natural, alternating square, and adjoint representations and the only absolutely irreducible representations of dimension less than $n^{3}$ in the symplectic and orthogonal cases are the trivial, natural, and alternating square representations. The extremely primitive groups obtained from the natural representations are listed in Theorems 1.2 (b) and 1.3 (c), and by Lemmas 4.5 and 4.6, the alternating square and adjoint representations also lead only to extremely primitive permutation groups listed in these theorems. If $n \leq 16$ in the special linear and unitary cases, or $n \leq 11$ in the symplectic and orthogonal cases, then the representations of $\operatorname{Soc}(H)$ of dimension less than $d_{1}(H)$ are listed in [21]. We listed the representations of dimension $d$ with $20<d<d_{1}(H)$ in the statement of Theorem 4.8 as candidates leading to extremely primitive examples. The remaining small-dimensional cases (excluding the natural, alternating square and adjoint representations, and their duals) are $(d, \operatorname{Soc}(H))=(20, \operatorname{SL}(4,2)),(20, \operatorname{SL}(6,2)),(20, \operatorname{PSU}(6,2)),\left(16, \Omega^{+}(10,2)\right)$, and the symplectic cases $(8, \operatorname{Sp}(6,2))$ and $(16, \operatorname{Sp}(8,2))$. In these cases, we constructed the $H$-module using atlasrep and computed all orbit lengths of $H$. The only case when $H$ acts primitively on all of its orbits is $(d, H)=(8, \operatorname{Sp}(6,2))$ (listed in Theorem $1.3(\mathrm{c}))$.

Similarly, if $H$ is exceptional then all representations of dimension less than $d_{1}(H):=2\lceil\log |H|\rceil$ are listed in [21]. The representations of dimension $d$ with $26<d<d_{1}(H)$ are listed in the statement of Theorem 4.8. The remaining cases are $(d, \operatorname{Soc}(H))=\left(14, G_{2}(2)^{\prime}\right),\left(26,{ }^{3} D_{4}(2)\right),\left(26, F_{4}(2)\right)$, and $\left(26,{ }^{2} F_{4}(2)^{\prime}\right)$. In all of these cases, the $H$-modules are available in atlasrep. In the case where $(d, \operatorname{Soc}(H))=\left(14, G_{2}(2)^{\prime}\right)$, we computed the lengths of all $H$-orbits and verified that $H$ does not act primitively on all of them. The 26-dimensional modules are too big for the computation of all $H$-orbits. In the cases $H={ }^{3} D_{4}(2)$ and ${ }^{3} D_{4}(2) .3$, elements of order 28 have two-dimensional fixed point spaces. Two of the fixed vectors are in $H$-orbits of length $72 \times 819$ so their stabilizer is not maximal in $H$. In the case $H=F_{4}(2)$, elements of order 30 have two-dimensional fixed point spaces. One of the fixed vectors is in an $H$-orbit of length $256 \times 69615$ so its stabilizer is not maximal in $H$. In the cases $H={ }^{2} F_{4}(2)^{\prime}$ and ${ }^{2} F_{4}(2)$, elements of order 16 have two-dimensional fixed point spaces and at least two fixed vectors are in $H$-orbits of length $80 \times 1755$ so their stabilizers are not maximal in $H$. 


\section{References}

[1] M. Aschbacher, On the maximal subgroups of the finite classical groups. Invent. Math. 76 (1984), 469-514. Zbl 0537.20023 MR 746539

[2] P. J. Cameron, Permutation Groups. London Math. Soc. Stud. Texts 45, Cambridge University Press, Cambridge 1999. Zbl 0922.20003 MR 1721031

[3] L. E. Dickson, Linear groups with an exposition of the Galois field theory. B.G. Teubner, Leipzig 1901; Dover, New York 1958. Zbl 0082.24901 MR 104735

[4] J. D. Dixon and B. Mortimer, Permutation groups. Grad. Texts in Math. 163, SpringerVerlag, New York 1996. Zbl 0951.20001 MR 1409812

[5] W. Feit and G. M. Seitz, On finite rational groups and related topics. Illinois J. Math. 33 (1989), 103-131. Zbl 0701.20005 MR 974014

[6] The GAP Group, GAP - Groups, algorithms, and programming, Version 4.4.9, 2006. http://www.gap-system.org

[7] D. Gorenstein, Finite groups the centralizers of whose involutions have normal 2-complements. Canad. J. Math. 21 (1969), 335-357. Zbl 0201.03202 MR 0242939

[8] R. M. Guralnick, K. Magaard, J. Saxl, and P. H. Tiep, Cross characteristic representations of symplectic and unitary groups. J. Algebra 257 (2002), 291-347. Zbl 1025.20002 MR 1947325

[9] J. E. Humphreys, Modular representations of finite groups of Lie type. In Finite Simple Groups II (ed. M. J. Collins), Academic Press, London 1980, 259-290. Zbl 0472.20015 MR 0606048

[10] G. D. James, On the minimal dimensions of irreducible representations of symmetric groups. Math. Proc. Cambridge Philos. Soc. 94 (1983), 417-424. Zbl 0544.20011 MR 720791

[11] C. Jansen, The minimal degrees of faithful representations of the sporadic simple groups and their covering groups. LMS J. Comput. Math. 8 (2005), 122-144. Zbl 1089.20006 MR 2153793

[12] C. Jansen, K. Lux, R. Parker, and R. Wilson, An atlas of Brauer characters. Clarendon Press, Oxford 1995. Zbl 0831.20001 MR 1367961

[13] P. Kleidman and M. W. Liebeck, The subgroup structure of the finite classical groups. London Math. Soc. Lecture Note Ser. 129, Cambridge University Press, Cambridge 1990. Zbl 0697.20004 MR 1057341

[14] L. G. Kovács, Primitive subgroups of wreath products in product action. Proc. London Math. Soc. (3) 58 (1989), 306-322. Zbl 0671.20002 MR 977479

[15] V. Landazuri and G. M. Seitz, On the minimal degrees of projective representations of the finite Chevalley groups. J. Algebra 32 (1974), 418-443. Zbl 0325.20008 MR 0360852

[16] M. W. Liebeck, On the orders of maximal subgroups of the finite classical groups. Proc. London Math. Soc. (3) 50 (1985), 426-446. Zbl 0591.20021 MR 779398

[17] M. W. Liebeck, The affine permutation groups of rank three. Proc. London Math. Soc. (3) 54 (1987), 477-516. Zbl 0621.20001 MR 879395 
[18] M. W. Liebeck, B. M. S. Martin, and A. Shalev, On conjugacy classes of maximal subgroups of finite simple groups, and a related zeta function. Duke Math. J. 128 (2005), 541-557. Zbl 1103.20010 MR 2145743

[19] M. W. Liebeck, C. E. Praeger and J. Saxl, On the O'Nan-Scott Theorem for finite primitive permutation groups. J. Austral. Math. Soc. Ser. A 44 (1988), 389-396. Zbl 0647.20005 MR 929529

[20] M. W. Liebeck and A. Shalev, Maximal subgroups of symmetric groups. J. Combin. Theory Ser. A 75 (1996), 341-352. Zbl 0866.20003 MR 1401008

[21] F. Lübeck, Small degree representations of finite Chevalley groups in defining characteristic. LMS J. Comput. Math. 4 (2001), 135-169. Zbl 1053.20008 MR 1901354

[22] W. A. Manning, Simply transitive primitive groups. Trans. Amer. Math. Soc. 29 (1927), 815-825. JFM 53.0108.01 MR 1501415

[23] D. V. Pasechnik and C. E. Praeger, On transitive permutation groups with primitive subconstituents. Bull. London Math. Soc. 31 (1999), 257-268. Zbl 0940.20004 MR 1673405

[24] D. S. Passman, Solvable 3/2-transitive permutation groups. J. Algebra 7 (1967), 192-207. Zbl 0244.20005 MR 0235018

[25] D. S. Passman, Exceptional 3/2-transitive permutation groups. Pacific J. Math. 29 (1969), 669-713. Zbl 0177.03701 MR 0244363

[26] G. Schlichting, Operationen mit periodischen Stabilisatoren. Arch. Math. 34 (1980), 97-99. Zbl 0449.20004 MR 583752

[27] G. M. Seitz and A. E. Zalesskii, On the minimal degrees of projective representations of the finite Chevalley groups, II. J. Algebra 158(1993), 233-243. Zbl 0789.20014 MR 1223676

[28] M. Suzuki, On a finite group with a partition. Arch. Math. 12 (1961), 241-254. Zbl 0107.25902 MR 0136647

[29] P. H. Tiep, Low dimensional representations of finite quasisimple groups. In Groups, combinatorics \& geometry (Durham, 2001), World Scientific, Singapore 2003, 277-294. Zbl 1032.20008 MR 1994973

[30] H. Wielandt, Finite permutation groups. Academic Press, New York 1964.Zbl 0138.02501 MR 183775

[31] R. A. Wilson, R. A. Parker, J. Bray, and T. Breuer, AtlasRep, a GAP package, version 1.3. http://www.math.rwth-aachen.de/ Thomas.Breuer/atlasrep/

[32] R. A. Wilson et al., ATLAS of finite group representations, version 3. http://brauer.maths.qmul.ac.uk/Atlas/v3/

Received February 6, 2007; revised March 26, 2007

A. Mann, Einstein Institute of Mathematics Givat Ram, The Hebrew University of Jerusalem, Jerusalem, 91904, Israel

E-mail: mann@math.huji.ac.il 
C. E. Praeger, School of Mathematics and Statistics, The University of Western Australia, 35 Stirling Highway, Crawley, WA 6009, Australia

E-mail: praeger@maths.uwa.edu.au

Á. Seress, Department of Mathematics, The Ohio State University, Columbus, Ohio 43210, U.S.A.

E-mail: akos@math.ohio-state.edu 